\title{
Spin Polarization Oscillations without Spin Precession: Spin-Orbit Entangled Resonances in Quasi-One-Dimensional Spin Transport
}

\author{
D. H. Berman, M. Khodas, and M.E. Flatté \\ Department of Physics and Astronomy, University of Iowa, Iowa City, Iowa 52242, USA
}

(Received 17 September 2013; published 25 March 2014)

\begin{abstract}
Resonant behavior involving spin-orbit entangled states occurs for spin transport along a narrow channel defined in a two-dimensional electron gas, including an apparent rapid relaxation of the spin polarization for special values of the channel width and applied magnetic field (so-called ballistic spin resonance). A fully quantum-mechanical theory for transport using multiple subbands of the one-dimensional system provides the dependence of the spin density on the applied magnetic field and channel width and position along the channel. We show how the spatially nonoscillating part of the spin density vanishes when the Zeeman energy matches the subband energy splittings. The resonance phenomenon persists in the presence of disorder.
\end{abstract}

DOI: 10.1103/PhysRevX.4.011048

\section{INTRODUCTION}

The properties of spin transport in small nonmagnetic structures determine whether it is favorable to scale down certain semiconductor spintronic devices [1-6] to the nanoscale. Spin-orbit (SO) fields, originating from a material's electronic structure [7], govern spin-transport phenomena in most nonmagnetic materials. These SO fields in nonmagnetic semiconductors cause a carrier's spin to precess around an axis with a rate that depends strongly on that carrier's momentum, an effect that produces coherent spin precession even during diffusive transport [8-10]. The spin of each carrier also dephases relative to other carriers with different momenta; as the carriers scatter from one momentum state to another, this dephasing results in decoherence through randomization of the carrier momentum [7,11-13]. Confinement of the carrier wave function to a smaller region, or application of a magnetic field [14,15], can quench the carrier momentum along one or more axes, reducing the random character of the SO field. Thus confinement of carriers in a semiconductor into a one-dimensional channel was predicted to lengthen the spin coherence time [16-19]. These longer spin coherence times even occur for channels much wider than the mean-free path [20], due to an interplay between the SO field and disorder in the confined geometry $[21,22]$ that yields long-lived spin spirals [21-24]. An inplane magnetic field can also modify the effect of the SO field and lengthen the spin coherence time [23,25]. With both confinement and in-plane magnetic fields present,

Published by the American Physical Society under the terms of the Creative Commons Attribution 3.0 License. Further distribution of this work must maintain attribution to the author(s) and the published article's title, journal citation, and DOI.
Subject Areas: Spintronics

however, an apparent reduction in the spin coherence length has been observed [26]. Reference [26] introduced a semiclassical model, further expanded upon in Ref. [27], suggesting that when the carrier transit time was similar to the spin precession time, a so-called ballistic spin resonance (BSR) would reduce the spin coherence length.

Here the phenomenon of ballistic spin resonance is placed on more solid theoretical ground using a quantum-mechanical microscopic theory of spin transport in a channel within which multiple subbands are occupied. The feature identified as BSR in Ref. [26] originates from coupling, via the SO interaction, between pairs of subbands that differ in both spin and orbital quantum numbers, yielding coherent precession of a "pseudospin." The magnetic-field dependence of the resulting propagating spin polarization is calculated assuming the spins are injected into the channel uniformly across the width of the channel. The pseudospin precession then yields spatial oscillations of the spin polarization as the distance along the channel from the injection location increases, and a transverse spin texture with no net transverse spin polarization. In Ref. [26], the detector was insensitive to the transverse spin polarization, and was set a fixed distance from the injection point; for such a geometry, the BSR phenomenon manifests as an apparent reduction of the spin coherence length. We consider the effect of disorder on the propagation and find that the predicted features of spin transport in a narrow channel are robust. Greater spatial resolution in the spin detection process would allow the oscillations to be directly imaged, and could permit them to be used in processing spin information within a small semiconductor device, or guiding it along a controllable pathway within a semiconductor chip.

This paper first treats, in Sec. II, the essential qualitative features of the BSR phenomenon in the clean limit, based 
on a two-subband toy model that illustrates the key features: pseudospin precession, spin polarization oscillation, and transverse spin texture. These results are then placed on a firm footing in Sec. III for the general case of multiple transverse subbands, and comparison is made with the results of Ref. [26]. The effect of disorder on these phenomena is analyzed in Sec. IV, and the key features are found to be robust. Some comments about other effects, such as the nature of the spin injection at a quantum point contact, are made in concluding remarks in the final section, Sec. V. Some useful relations relevant for the boundary conditions, including spin injection and detection, are derived in the Appendix.

\section{QUALITATIVE PICTURE OF BALLISTIC SPIN RESONANCE}

Figure 1 is a schematic of Ref. [26]. A channel in a twodimensional electron gas has width $W$ in the $\hat{z}$ direction and extends along the $\hat{x}$ direction. The external applied magnetic field is oriented along $\hat{z}$ so that spins polarized along $\hat{z}$ are injected and detected by two quantum point contacts (QPC) located at $x_{s}$ and $x_{d}$.

A simple two-subband model with dispersion relations shown in Fig. 2(a) illustrates the essential aspects of the variation of spin density with distance. The two subbands are labeled 1 and 2. The doubly degenerate spin states associated with each subband are split by the Zeeman interaction with a magnetic field oriented along the $\hat{z}$ direction. Once the SO interaction is added, subband 1 with spin-down is coupled to subband 2 with spin-up. This results in two eigenstates, shown in Fig. 2(b), with different energies (upper and lower) that consist of a mixture of up and down spins and the two subband states (orbital motion). When a spin density is injected into the system at the Fermi energy $E_{F}$, instead of going into unoccupied pure spin eigenstates at the Fermi energy, that spin must occupy linear superpositions of the two mixed eigenstates, shown in Fig. 2(b), which propagate with different wave vectors $k_{+}$and $k_{-}$down the channel, as shown. The beating

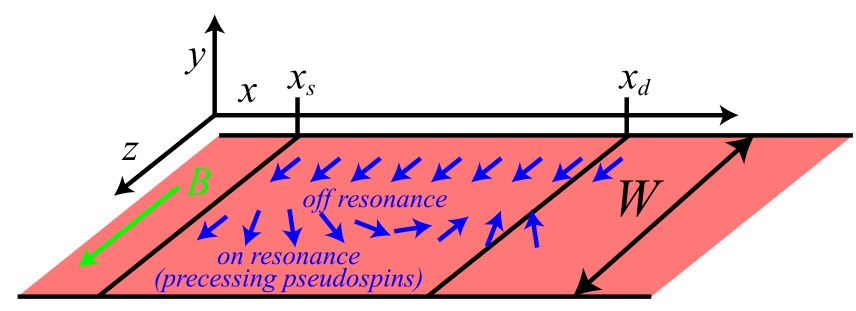

FIG. 1. Schematic representation of the experimental setup of Ref. [26]. A quasi-one-dimensional channel of width $W$ is defined by confinement of a two-dimensional electron gas in the $x z$ plane. The in-plane magnetic field is applied in the $\hat{z}$ direction. $x_{s}$ and $x_{d}$ denote the location of the source and drain of spin polarization along the channel.

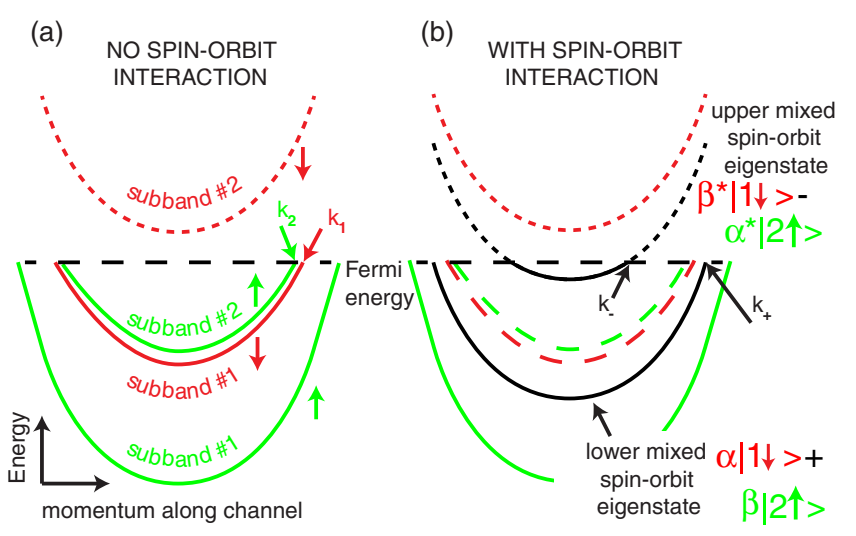

FIG. 2. The effect of SO coupling illustrated for two subbands (1 and 2) with different lateral orbital wave functions. (a) Without SO the in-plane magnetic field splits each subband into spin-up and spin-down subbands separated by the Zeeman energy $E_{Z}$. At the resonance condition, Eq. (21), the spin-down states of subband 1 coincide with the spin-up states of subband 2. (b) With SO these states mix, and the SO-coupling-induced mixing is strongest exactly at the resonance condition, where $|\alpha|=|\beta|=1 / \sqrt{2}$.

of these wave vectors gives rise to a spatial oscillation of spin density.

Consider Fig. 2 in more detail. Without spin-orbit interaction, but with a Zeeman interaction, states in the waveguide are of the form

$$
\psi_{k, n, s}^{Z}=e^{i k x} \phi_{n}(z)|s\rangle
$$

where the index $n$ enumerates subbands of transverse quantization and $\sigma_{z}|s\rangle=s|s\rangle$, with $s= \pm 1$ corresponding to spin-up $|\uparrow\rangle$ or spin-down $|\downarrow\rangle$ spin polarization. Here we consider only the two subbands $n=1,2$. The energies of Zeeman-split states, Eq. (1), can be written as

$$
E_{k, n, s}^{Z}=\frac{\hbar^{2} k^{2}}{2 m}+\epsilon_{n, s}^{Z}
$$

where the first term is the kinetic energy of motion along the channel, and the second term

$$
\epsilon_{n, s}^{Z}=\epsilon_{n}-s \frac{E_{Z}}{2}
$$

includes the energy of transverse quantization $\epsilon_{n}$ and the Zeeman energy $-s E_{Z} / 2$. The negative sign in Eq. (3) corresponds to a negative $g$ factor, and the spin splitting is related to the magnetic field $\boldsymbol{B}$ in a standard way, $E_{Z}=|g| \mu_{B} B$. The two Zeeman-split subbands are shown in Fig. 2(a).

The states relevant to dc transport have energies close to the Fermi level. Hence, we determine the Fermi wave vectors of the states at the Fermi energy $E_{F}$, shown in Fig. 2(a), by solving $E_{F}=E_{k, n, s}^{Z}$. The pair of adjacent 
subbands $\psi_{k, 1, \downarrow}^{Z}$ and $\psi_{k, 2, \uparrow}^{Z}$ is critical for the BSR. For these two subbands, the Fermi wave vectors $k_{1}$ and $k_{2}$ are

$$
k_{1}=\sqrt{\frac{2 m}{\hbar^{2}}\left(E_{F}-\epsilon_{1, \downarrow}^{Z}\right)}
$$

and

$$
k_{2}=\sqrt{\frac{2 m}{\hbar^{2}}\left(E_{F}-\epsilon_{2, \uparrow}^{Z}\right)},
$$

with the energy $\epsilon_{n, s}^{Z}$ introduced in Eq. (3).

\section{A. Subband mixing by SO coupling}

We now consider the effect of the SO coupling on the states [Eq. (1)] and energies [Eqs. (2) and (3)] within the degenerate perturbation theory applicable if the typical SO interaction energy is smaller than intersubband separation. Specifically, we focus on a SO coupling of the form

$$
H_{\mathrm{SO}}=\alpha_{-} p_{z} \sigma_{x}
$$

More general spin-orbit terms in the Hamiltonian will be discussed in later sections of the paper. Under the condition of BSR, the states $\psi_{k, 2, \uparrow}^{Z}$ and $\psi_{k, 1, \downarrow}^{Z}$ are near degeneracy at each $k$ and are coupled by this SO coupling because the matrix element $\left\langle 2, \uparrow\left|\alpha_{-} p_{z} \sigma_{x}\right| 1, \downarrow\right\rangle=i \delta^{\mathrm{SO}} / 2$ is, in general, nonzero (here $\delta^{\mathrm{SO}}$ is real). The spectrum and eigenstates are found by diagonalizing the projection $\mathrm{H}_{2}$ of the full Hamiltonian onto the subspace spanned by these two states,

$$
H_{2}(k)=\frac{\hbar^{2} k^{2}}{2 m}-\left[\begin{array}{cc}
E_{Z} / 2-\epsilon_{2} & -i \delta^{\mathrm{SO}} / 2 \\
i \delta^{\mathrm{SO}} / 2 & -E_{Z} / 2-\epsilon_{1}
\end{array}\right] .
$$

The eigenstates, obtained by a straightforward diagonalization of Eq. (7), are denoted by $\psi_{+}^{\mathrm{SO}}$ and $\psi_{-}^{\mathrm{SO}}$ at a given wave vector $k$, and are linear combinations of the states defined in Eq. (1) [see Fig. 2(b)],

$$
\begin{aligned}
& \left|\psi_{k,+}^{\mathrm{SO}}\right\rangle=\alpha\left|\psi_{k, 2, \uparrow}^{Z}\right\rangle+\beta\left|\psi_{k, 1, \downarrow}^{Z}\right\rangle, \\
& \left|\psi_{k,-}^{\mathrm{SO}}\right\rangle=\beta^{*}\left|\psi_{k, 2, \uparrow}^{Z}\right\rangle-\alpha^{*}\left|\psi_{k, 1, \downarrow}^{Z}\right\rangle,
\end{aligned}
$$

where

$$
\alpha=i \cos (\theta / 2), \quad \beta=-\sin (\theta / 2) .
$$

The angle $\theta$ parametrizes the matrix, Eq. (7), through

$$
\begin{aligned}
& \cos \theta=\frac{E_{Z}-\Delta E}{\sqrt{\left(E_{Z}-\Delta E\right)^{2}+\left(\delta^{\mathrm{SO}}\right)^{2}}}, \\
& \sin \theta=\frac{\delta^{\mathrm{SO}}}{\sqrt{\left(E_{Z}-\Delta E\right)^{2}+\left(\delta^{\mathrm{SO}}\right)^{2}}},
\end{aligned}
$$

where the intersubband splitting in the absence of SO interaction is $\Delta E=\epsilon_{2}-\epsilon_{1}$. The energies of the eigenstates in Eq. (8) are

$$
\begin{aligned}
E_{k, \pm}^{\mathrm{SO}} & =\frac{\hbar^{2} k^{2}}{2 m}+\epsilon_{ \pm}^{\mathrm{SO}} \\
\epsilon_{ \pm}^{\mathrm{SO}} & =\frac{1}{2}\left(\epsilon_{1}+\epsilon_{2}\right) \mp \frac{1}{2} \sqrt{\left(E_{Z}-\Delta E\right)^{2}+\left(\delta^{\mathrm{SO}}\right)^{2}} .
\end{aligned}
$$

The band splitting

$$
\epsilon_{-}^{\mathrm{SO}}-\epsilon_{+}^{\mathrm{SO}}=\sqrt{\left(E_{Z}-\Delta E\right)^{2}+\left(\delta^{\mathrm{SO}}\right)^{2}}
$$

is nonzero, even when the bands overlap in the absence of SO coupling, $E_{Z}=\Delta E$; see Fig. 2(b). Equations (8)-(11) specify the mixing effect of the SO coupling on the adjacent subbands shown schematically in Fig. 2.

The Fermi wave vectors $k_{ \pm}$of the two subbands with the dispersion relations of Eq. (11), shown in Fig. 2(b), satisfy $E_{F}=E_{k_{ \pm}, \pm}^{\mathrm{SO}}$, and are

$$
k_{ \pm}=\sqrt{\frac{2 m}{\hbar^{2}}\left(E_{F}-\epsilon_{ \pm}^{\mathrm{SO}}\right)} .
$$

\section{B. Spatial dependence of spin polarization along the channel}

Now we follow the spatial evolution of the states injected into the channel at $x_{s}$ at the Fermi level, as shown in Fig. 1. The spinor wave function of the injected electron $|\Psi\rangle_{\text {inj }}$ is a superposition of the eigenstates given by Eq. (8). We can write this spinor as

$$
|\Psi\rangle_{\mathrm{inj}}=c_{+}\left|\psi_{k_{+},+}^{\mathrm{SO}}\right\rangle+c_{-}\left|\psi_{k_{-}-}^{\mathrm{SO}}\right\rangle,
$$

where the Fermi wave vectors of the two SO split states are given by Eq. (13) [see Fig. 2(b)]. If the injected spin is polarized along the $z$ direction, the coefficients $c_{ \pm}$are, up to an overall phase factor, $c_{+}=\alpha^{*}$ and $c_{-}=\beta$, where $\alpha$ and $\beta$ are defined in Eqs. (9) and (10). The spin polarization $\tilde{s}_{z}(x, z)=\left\langle\Psi\left|\sigma_{z}\right| \Psi\right\rangle$ can be written using the explicit expressions in Eqs. (1), (8), and (14) as follows:

$$
\begin{aligned}
\tilde{s}_{z}(x, z)= & {\left[|\alpha|^{2}-|\beta|^{2}\right]\left[|\alpha|^{2} \phi_{1}^{2}(z)-|\beta|^{2} \phi_{2}^{2}(z)\right] } \\
& +2|\alpha|^{2}|\beta|^{2}\left[\phi_{1}^{2}(z)\right. \\
& \left.+\phi_{2}^{2}(z)\right] \cos \left[\left(k_{+}-k_{-}\right)\left(x-x_{s}\right)\right] .
\end{aligned}
$$

The spin polarization per unit length,

$$
s_{z}(x)=\int d z \tilde{s}_{z}(x, z)
$$

which is obtained from Eqs. (9) and (15), reduces to a simple expression that illustrates many of the key features of BSR: 


$$
s_{z}(x, B)=\cos ^{2} \theta+\sin ^{2} \theta \cos \left[\left(k_{+}-k_{-}\right)\left(x-x_{s}\right)\right] .
$$

We refer to the part of $s_{z}(x, B)$ that does not oscillate with $x$ as the conserved part of the spin density. The oscillation of the spin density is a result of the spatial beating of the two wave functions $\left|\psi_{k_{+}, \pm}^{\mathrm{SO}}\right\rangle$, which propagate along the waveguide with phases $\exp \left[i k_{ \pm}\left(x-x_{s}\right)\right]$.

A key feature of BSR, which produces the apparent reduction in spin coherence length, is that the conserved part of the spin density vanishes when the level separation equals the Zeeman splitting, $\Delta E=E_{Z}$. Furthermore, when many bands cross the Fermi level or when there is intersubband scattering there will be many sinusoidal contributions to the spin density like those in Eq. (17), but associated with differing wave vector differences, $k_{+}-k_{-}$. When added together these will tend to cancel one another. As a result, when the resonance condition $\Delta E=E_{Z}$ holds, not only will the constant (conserved) part of the spin density vanish, but also the oscillating (nonconserved) part will be small. Therefore, the total spin density will be small at resonance.

The above picture of the BSR, based on a separation of conserved and nonconserved parts (constant and oscillatory in space, respectively), can be illustrated by writing the effective Hamiltonian, Eq. (7) in the form

$$
H_{2}(k)=\left[\frac{\hbar^{2} k^{2}}{2 m}+\frac{\epsilon_{1}+\epsilon_{2}}{2}\right]-\frac{1}{2}\left|g \mu_{B}\right| \mathbf{B}_{\text {eff }} \cdot \boldsymbol{\tau}
$$

where $\tau_{x, y, z}$ are pseudospin Pauli matrices. The effective field points in the direction $\hat{e}=(0, \sin \theta, \cos \theta)$ and the spins precess about this field as they propagate down the channel.

The effective field in the $y$ direction,

$$
B_{\mathrm{eff}, y}=\left\langle n+1, \uparrow\left|\alpha_{-} p_{z} \sigma_{x}\right| n, \downarrow\right\rangle,
$$

arises from SO matrix elements between states of differing spin and orbital quantum numbers (in the absence of SO). The effective magnetic field in the $z$ direction accounts for the energy splitting between adjacent modes arising both from confinement $(\Delta E)$ and from the Zeeman interaction with the actual external field $B_{z}$,

$$
B_{\mathrm{eff}, z}=B_{z}-\Delta E /\left(g \mu_{B}\right) .
$$

BSR then occurs when $B_{\mathrm{eff}, z}=0$, corresponding to

$$
g\left|\mu_{B}\right| B_{z} \equiv E_{Z}=\Delta E,
$$

and the effective field lies entirely along the $\hat{y}$ axis, so spins in the $z$ direction have no nonprecessing part, as schematically indicated in Fig. 3. For square-well confinement, only a few modes near cutoff can be in resonance for a given external field $B$; for parabolic confinement, adjacent modes at $B=0$ are all separated by a single energy $\hbar \omega=\Delta E$, so

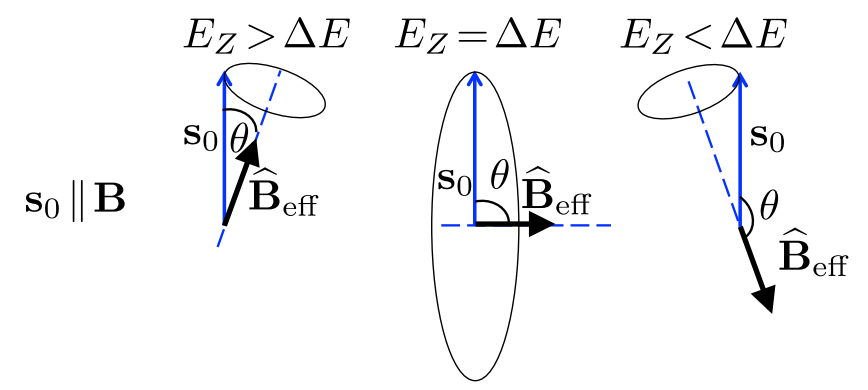

FIG. 3. The injected spin polarization $s_{0}$ is directed along the external in-plane magnetic field $\mathbf{B} \| \hat{z}$; see Fig. 1 . The effective magnetic field $\mathbf{B}_{\text {eff }}$ determines the pseudospin precession after injection. The $z$ component of $\mathbf{B}_{\text {eff }}$, Eq. (20), vanishes at the resonance condition, Eq. (21). At resonance, the pseudospin dynamics is a pure precession with a frequency controlled by the SO coupling matrix element, $\left(g \mu_{B} / \hbar\right)\left|\mathbf{B}_{\text {eff }}\right|=$ $(1 / \hbar)\left|\left\langle n+1, \uparrow\left|\alpha p_{z} \sigma_{x}\right| n, \downarrow\right\rangle\right|$. Off resonance, a finite component of injected spin $(\propto \cos \theta)$ is conserved and is represented by an $x$-independent part in Eq. (17).

that all pairs go into resonance for the same magnetic field. Equation (21) is the same condition for resonance as that cited in Ref. [26].

We note that the pseudospin precession around the effective field $\mathbf{B}_{\text {eff }}$ is not equivalent to spin precession. More precisely, the absence of a net spin precession is clearly indicated by the result that $s_{x, y}(x, B)=0$ everywhere along the channel. Despite no net spin precession, however, there is a definite spin texture evident transverse to the channel, for

$$
\tilde{s}_{x}(x, z, B) \propto \sin \left[\left(k_{+}-k_{-}\right)\left(x-x_{s}\right)\right] \phi_{1}(z) \phi_{2}(z),
$$

which is obtained similarly to Eq. (15). This transverse spin texture is missing in the theory of Refs. [26],[27].

In the next section, we derive a general expression for the spin density for multiple occupied subbands in the channel. The expressions we obtain are exact provided the spin-orbit Hamiltonian has the form of Eq. (6), and provided there is neither scattering nor electron-electron interaction; Eq. (17) is a special case of this expression. We further compare spin densities for parabolic and square-well confining potentials.

\section{THEORY OF SPIN POLARIZATION TRANSPORT IN A QUASI-ONE-DIMENSIONAL CHANNEL}

We now generalize to the case where multiple subbands are occupied, and derive a generalization of Eq. (17). Let the rate of the spin injection per unit length be $F(x)$. Then the stationary polarization is given by

$$
s_{z}(x, B)=-\left.i \int \frac{d q}{2 \pi} e^{i q x}\left[\partial_{\omega} \chi(q, \omega) \chi^{-1}(q)\right]\right|_{\omega \rightarrow i 0} F(q) .
$$


Equation (23) relates the spin density to the Fourier image of the injection rate $F(q)$ via the retarded correlation function

$$
\chi(q, \omega)=-i \int_{0}^{\infty} d t \int d x e^{-i q x+i \omega t}\left\langle\left[\hat{s}_{z}(x, t), \hat{s}_{z}(0,0)\right]\right\rangle
$$

of the spin density operator,

$$
\hat{S}_{z}(x)=\frac{1}{2} \int_{-\infty}^{+\infty} d z\left[\psi_{\uparrow}^{\dagger}(x, z) \psi_{\uparrow}(x, z)-\psi_{\downarrow}^{\dagger}(x, z) \psi_{\downarrow}(x, z)\right],
$$

where $\psi_{\uparrow, \downarrow}^{\dagger}(x, z)$ is the second quantized creation operator and the spin quantization axis $\hat{z}$ coincides with the direction of the in-plane magnetic field, which is perpendicular to the channel, as shown in Fig. 1. Equation (23) is derived in the Appendix, and its limitations are discussed in Sec. V.

We apply Eq. (23) to noninteracting electrons in a clean wire with parabolic or square-well confinement, with the Hamiltonian

$$
H=\frac{p_{x}^{2}+p_{z}^{2}}{2 m}+V_{c}(z)-\frac{1}{2} E_{Z} \sigma_{z}+H_{\mathrm{SO}}
$$

Here $V_{c}(z)$ is the lateral confinement potential, $E_{Z}$ is the Zeeman splitting, and $H_{\mathrm{SO}}=\alpha_{-} p_{z} \sigma_{x}+\alpha_{+} p_{x} \sigma_{z}$ is the $\mathrm{SO}$ interaction term with $\alpha_{+,(-)}$equal to the sum (difference) of the Rashba [28,29] and Dresselhaus [30] coefficients. As the term with $\alpha_{+}$is proportional to $\sigma_{z}$, it does not couple subbands with opposite spin direction along $\hat{z}$; its effect is entirely to provide a $k_{x}$-dependent shift of the Zeeman energy. Therefore, to simplify the calculations presented here, we neglect the effect of this term and set $\alpha_{+}=0$. We consider the effect of nonzero $\alpha_{+}$in Sec. IV C.

Without SO coupling, the eigenstates of the Hamiltonian, Eq. (26), can be denoted by their wave vector along the channel $k$, their spin $s$ (up or down) in the $z$ direction across the channel, and the natural mode numbers $m$ arising from confinement, as in the previous section:

$$
\left\langle x, z \mid \psi_{k, m, s}^{Z}\right\rangle=e^{i k x} \phi_{k, m}(z)|s\rangle=e^{i k x} \phi_{m}(z)|s\rangle .
$$

The eigenstates with SO coupling can be expanded in terms of these uncoupled states as

$$
\left|\psi_{k, n, \gamma}^{\mathrm{SO}}\right\rangle=\sum_{m, s} A_{m, s}^{n, \gamma}(k, B)\left|\psi_{k, m, s}^{Z}\right\rangle
$$

$(\gamma= \pm 1)$, with corresponding energies given by

$$
E_{k, n, \gamma}^{\mathrm{SO}}=\frac{\hbar^{2} k^{2}}{2 m}+\epsilon_{k, n, \gamma}^{\mathrm{SO}}
$$

If $\alpha_{+}=0$, the expansion coefficients $A_{m, s}^{n, \gamma}(k, B)$ do not depend on the momentum $k$ and the energies $\epsilon_{k, n, \gamma}^{\mathrm{SO}}$ do not depend on $k$, so $\epsilon_{k, n, \gamma}^{\mathrm{SO}}=\epsilon_{n, \gamma}^{\mathrm{SO}}$. The SO states can then be written as

$$
\left|\psi_{k, n, \gamma}^{\mathrm{SO}}\right\rangle=e^{i k x}|n, \gamma\rangle
$$

We stress that the eigenstates $\left|\psi_{k, n, \gamma}^{\text {sO }}\right\rangle$ in Eq. (28) are superpositions of Zeeman states with different subband index $m$ and spin index $s$ but with the same wave vector $k$. Note that from here on the superscript SO will be dropped since all energies and wave functions will be those including spin-orbit interactions. Thus, the quantized energies of transverse modes listed in Eq. (29) are simply $\epsilon_{n, \gamma}$ and

$$
E_{k, n, \gamma}=\frac{\hbar^{2} k^{2}}{2 m}+\epsilon_{n, \gamma}
$$

The retarded susceptibility is given in terms of the eigenstates and energies of the coupled Hamiltonian by

$$
\begin{aligned}
\chi(q, \omega)= & \frac{-1}{2 \pi} \sum_{n, \gamma, n^{\prime}, \gamma^{\prime}}\left|\left\langle n, \gamma\left|\sigma_{z}\right| n^{\prime}, \gamma^{\prime}\right\rangle\right|^{2} \\
& \times \int d k \frac{f\left(E_{k, n, \gamma}\right)-f\left(E_{k+q, n^{\prime}, \gamma^{\prime}}\right)}{\omega+i 0+\epsilon_{n, \gamma}-\epsilon_{n^{\prime}, \gamma^{\prime}}-\left(\frac{\hbar^{2}}{2 m}\right)\left(2 k q+q^{2}\right)} .
\end{aligned}
$$

The zero temperature Fermi function is $f(\epsilon)=\Theta(\mu-\epsilon)$, where $\Theta$ is the Heaviside function and $\mu$ is the chemical potential. The integration over $k$ can be performed analytically. The static susceptibility is then

$$
\begin{aligned}
\chi^{s}(q)= & \frac{m}{\pi \hbar^{2} q} \sum_{n, \gamma, n^{\prime}, \gamma^{\prime}}\left|\left\langle n, \gamma\left|\sigma_{z}\right| n^{\prime}, \gamma^{\prime}\right\rangle\right|^{2} \\
& \times \log \frac{\left|q+\left(k_{n, \gamma}+k_{n^{\prime}, \gamma^{\prime}}\right)\right|}{\left|q-\left(k_{n, \gamma}+k_{n^{\prime}, \gamma^{\prime}}\right)\right|} .
\end{aligned}
$$

When $q \rightarrow 0$,

$$
\chi^{s}(q=0)=\frac{2 m}{\pi \hbar^{2}} \sum_{n, \gamma, n^{\prime}, \gamma^{\prime}} \frac{\left|\left\langle n, \gamma\left|\sigma_{z}\right| n^{\prime}, \gamma^{\prime}\right\rangle\right|^{2}}{k_{n, \gamma}+k_{n^{\prime}, \gamma^{\prime}}},
$$

where

$$
k_{n, \gamma}=\sqrt{\left(2 m / \hbar^{2}\right)\left(\mu-\epsilon_{n, \gamma}\right)}
$$

are the wave numbers for propagation along the wire corresponding to $k_{ \pm}$in Sec. II. The sum over modes is restricted to those with real $k_{n, \gamma}$.

To compute the spin density, we also need the frequency derivative of the susceptibility. The integrals here can also be computed with the result 
$\left.\partial_{\omega} \chi(q, \omega+i \delta)\right|_{\omega=0}=\frac{i 2 m^{2}}{\hbar^{3}} \sum_{n, \gamma, n^{\prime}, \gamma^{\prime}}\left|\left\langle n, \gamma\left|\sigma_{z}\right| n^{\prime}, \gamma^{\prime}\right\rangle\right|^{2} \times\left[\frac{\delta\left(q+k_{n, \gamma}-k_{n^{\prime}, \gamma^{\prime}}\right)}{2 k_{n, \gamma} k_{n^{\prime}, \gamma^{\prime}}}+\frac{\delta\left(q+k_{n, \gamma}+k_{n^{\prime}, \gamma^{\prime}}\right)}{2 k_{n^{\prime}, \gamma^{\prime}}\left(k_{n, \gamma}+k_{n^{\prime}, \gamma^{\prime}}\right)}+\frac{\delta\left(q-k_{n, \gamma}-k_{n^{\prime}, \gamma^{\prime}}\right)}{2 k_{n^{\prime}, \gamma^{\prime}}\left(k_{n, \gamma}+k_{n^{\prime}, \gamma^{\prime}}\right)}\right]$.

Finally, we arrive at a fairly simple, yet exact, result for the spin density using Eqs. (23), (32), and (35),

$$
\begin{aligned}
s(x)= & s_{0} \frac{m^{2}}{2 \pi \hbar^{3}} \\
& \times \sum_{n, \gamma, n^{\prime}, \gamma^{\prime}} \frac{\left|\left\langle n, \gamma\left|\sigma_{z}\right| n^{\prime}, \gamma^{\prime}\right\rangle\right|^{2} \cos \left[\left(k_{n, \gamma}-k_{n^{\prime}, \gamma^{\prime}}\right)\left(x-x_{0}\right)\right]}{\chi^{S}\left(k_{n, \gamma}-k_{n^{\prime}, \gamma^{\prime}}\right)\left(k_{n, \gamma} k_{n^{\prime}, \gamma^{\prime}}\right)} .
\end{aligned}
$$

For a source located at $x=x_{0}$, we take $F(q)=s_{0} \exp \left(-i q x_{0}\right)$. In practice, the matrix elements are only large when $(n, \gamma)$ is "near" $\left(n^{\prime}, \gamma^{\prime}\right)$ and the SO splitting is small so that $k_{n, \gamma}-k_{n^{\prime}, \gamma^{\prime}}$ is small. Then no major error is incurred if in the static susceptibility we set $k_{n, \gamma}-k_{n^{\prime}, \gamma^{\prime}}=0$. It should be noted, too, that in going from Eq. (23) to Eq. (36), we made use of the fact that the static susceptibility diverges (logarithmically) when $q= \pm\left(k_{n, \gamma}+k_{n^{\prime}, \gamma^{\prime}}\right)$ for any pair $(n, \gamma)$ and $\left(n^{\prime}, \gamma^{\prime}\right)$, so that the corresponding $\delta$ functions in the derivative of $\chi$ do not contribute to the spin density. This result for the spin density is exact, depending only on the assumptions that the wire is clean, the particles interact only with the confining potential, spin-orbit field, and magnetic field, and $\alpha_{+}=0$. Diagonal terms in the double sum over states give a contribution to the spin density independent of the distance $x-x_{0}$, while off-diagonal contributions provide a sum of sinusoidal contributions with spatial beat frequencies $k_{n, \gamma}-k_{n^{\prime}, \gamma^{\prime}}$.

The result for the spin density cited at the outset can now be obtained for parabolic confinement with $V(z)=$ $m \omega^{2} z^{2} / 2$. Then the only nonzero matrix elements of $\sigma_{z}$ in the $\mathrm{SO}$ basis are

$$
\left|\left\langle n, \gamma\left|\sigma_{z}\right| n, \gamma\right\rangle\right|^{2}=\cos ^{2} \theta_{n}=\left(\frac{E_{Z}-\hbar \omega}{\Delta_{n}}\right)^{2}
$$

and

$$
\left|\left\langle n, \gamma\left|\sigma_{z}\right| n,-\gamma\right\rangle\right|^{2}=\sin ^{2} \theta_{n}=\left(\frac{\delta_{n}^{\mathrm{SO}}}{\Delta_{n}}\right)^{2},
$$

where the SO splitting is given by

$$
\delta_{n}^{\mathrm{SO}}=\alpha_{-} \sqrt{2(n+1) m \omega / \hbar}
$$

and the level splitting by

$$
\Delta_{n}=\sqrt{\left(\delta_{n}^{\mathrm{SO}}\right)^{2}+\left(E_{Z}-\hbar \omega\right)^{2}} .
$$

The simplicity of the result for parabolic confinement occurs in part because the momentum operator for the harmonic oscillator has matrix elements only between adjacent non-SO-coupled states.

For parabolic confinement, we then find

$$
\begin{aligned}
s_{z}(x, B)= & \frac{s_{0}}{2 \sum_{n} v_{n}^{-1}} \sum_{n} v_{n}^{-2}\left\{\cos ^{2} \theta_{n}\right. \\
& \left.+\sin ^{2} \theta_{n} \cos \left[\frac{2 \pi}{l_{n}}\left(x-x_{s}\right)\right]\right\},
\end{aligned}
$$

where the sum runs over pairs of propagating modes, and $2 \pi l_{n}^{-1}=\left(k_{n, 1}-k_{n,-1}\right)$ is $2 \pi$ times the inverse spin precession length of an electron in the $n$th modes. $k_{n 1}$ and $k_{n,-1}$ have been replaced with $\left(k_{n, 1}+k_{n,-1}\right) / 2=m v_{n} / \hbar$ except in the term oscillating with $x-x_{s}$. The result for a single mode is Eq. (17). For other confining potentials, such as an infinite square well, eigenstates and energies can be found by diagonalizing a matrix with dimensions only slightly larger than the number of propagating modes.

The dependence of the off-diagonal matrix elements on the magnetic field is shown in Fig. 4. For parabolic confinement, modes at $B=0$ without SO splitting are equally spaced in energy; for a square well, the energy levels are spaced proportionally to $2 n+1$, where $n$ is the mode number. Ballistic spin resonance for a mode (spin) pair occurs when the Zeeman splitting is equal to the $B=0$ splitting. Thus, in the parabolic case, all modes can become resonant, as indicated by the black diamonds in the lefthand panel of Fig. 4. In the square-well case, it is not possible for all modes to become resonant for a single value of $B$. Furthermore, in the square well, modes that become resonant, but are located far away from the chemical potential $\mu$, produce small effects on the spin polarization versus $B$ curves (see Fig. 5); the dominant features of the spin resonance are determined by the spin-split mode pair with energies closest to $\mu$, i.e., the modes with the smallest $k_{n, \gamma}$. These modes make the largest contribution to the spin density.

The essence of the BSR is that the effective field becomes orthogonal to the injected spin polarization; i.e., $\cos \theta_{n}=0$. Any phase randomization (randomization of the $k_{n, \gamma}$ ) will then cause decay of the polarization away from the injector. The behavior of the spin density without phase randomization is shown as a function of $B$ and as a 


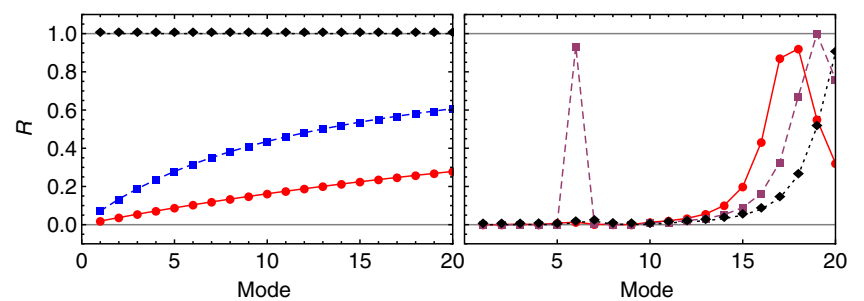

FIG. 4. Ratio of adjacent energy level splitting due to SO interaction to the total energy level splitting for pairs of propagating modes, $R=\left|\left\langle n, \gamma\left|\hat{s}_{z}\right| n,-\gamma\right\rangle\right|^{2} /\left(\left|\left\langle n, \gamma\left|\hat{s}_{z}\right| n, \gamma\right\rangle\right|^{2}+\right.$ $\left.\left|\left\langle n, \gamma\left|\hat{s}_{z}\right| n,-\gamma\right\rangle\right|^{2}\right)$. Parameters are chosen so that $B_{\text {resonant }}=$ $7 \mathrm{~T}$ and so that there are 20 propagating modes, $\mathrm{W}=1.15 \mu \mathrm{m}$. In the parabolic case, $R$ is just $\sin ^{2} \theta_{n}$. The lefthand panel shows $R$ for parabolic confinement; the right-hand panel shows $R$ for square-well confinement. $B=3 \mathrm{~T}$ (solid red line), $B=6 \mathrm{~T}$ (dashed blue line), and $B=7 \mathrm{~T}$ (dotted black line).
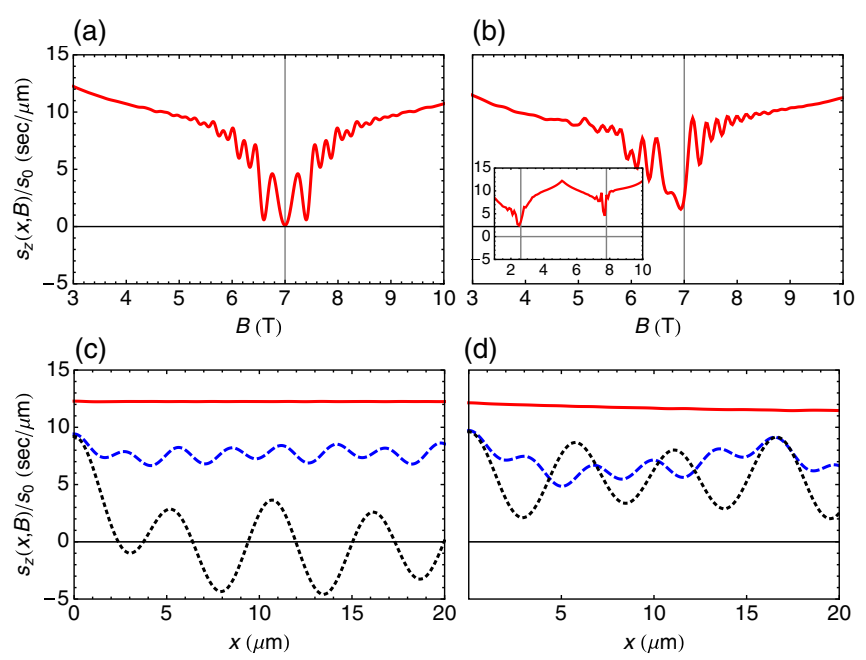

(d)

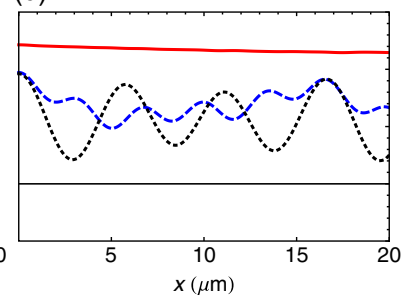

FIG. 5. The stationary spin polarization $s_{z}$ as a function of the magnetic field $B$ for (a) parabolic and (b) square-well lateral confinement. In all computations, $\alpha_{-}=2 \times 10^{-13} \mathrm{eV} \mathrm{m}$, and the width of the injection aperture is $0.5 \mu \mathrm{m}$. The effective mass is $m=0.067 m_{e}$, and the $g$ factor is -0.44 . The distance from the source, $x-x_{s}$, is $20 \mu \mathrm{m}$ in (a) and (b). For square-well confinement, the width of the well $W$ in (b) and (d) is $1.15 \mu \mathrm{m}$ The inset in (b) shows $s$ versus $B$ when $W=3 \mu \mathrm{m}$ and $x=6.7 \mu \mathrm{m}$, as in Ref. [26]. Except for the inset, the number of propagating modes (including spin) in all panels is 40. In the inset there are 105 propagating modes. Panels (c) and (d) show the dependence of the spin polarization on distance from the source for the parabolic and square-well lateral confinement, respectively. $B=3 \mathrm{~T}$ (solid red line), $B=6 \mathrm{~T}$ (dashed blue line), and $B=7 \mathrm{~T}$ (dotted black line). The parameters used for parabolic confinement are $\mu=3.65 \mathrm{meV}, \hbar \omega=$ $0.1785 \mathrm{meV}$, so that the magnetic field at the minimum is $7.0 \mathrm{~T}$. For square-well confinement, $\mu=1.86 \mathrm{meV}$, whereas in the inset it is $1.765 \mathrm{meV}$. Parameters have been chosen to mimic those of Ref. [26]. function of $x$ in Fig. 5, for parabolic and square-well confinement. The shape of the resonance dip in Figs. 5(a) and 5(b) depends on the nature of the confining potential. It also depends on how close the modes are to cutoff, i.e., on how small the $k_{n, \gamma}$ become. In these figures, we plot $s_{z}$ in a range of $B$ so that the uppermost modes are bounded away from cutoff. The impedance for injecting spins at the cutoff condition for a propagating mode must vanish, in analogy with total internal reflection in optics. The depth of the resonant dip depends on distance from the injection location $x-x_{s}$ as is shown in Figs. 5(c) and 5(d). For both confinements, $s_{z}$ versus $x$ seems to be a sum of a small number of oscillating terms, indicating that only a few modes contribute to the resonant dips in Figs. 5(a) and 5(b). This has been verified (but is not shown here) by simply calculating $s_{z}$ using only the two modes closest to cutoff.

The inset in Fig. 5 shows $s$ versus $B$ having two minima. The parameters have been chosen to mimic Fig. 2(b) of Ref. [26]. The first minimum comes from the condition $g\left|\mu_{B}\right| B=E_{m+1}-E_{m}=\Delta E_{m}$. In the case of parabolic confinement, only neighboring levels are coupled by the $\mathrm{SO}$ interaction. In the case of square-well confinement, SO interactions can couple modes of the form (now the indices refer to states not coupled by SO) $|m, s\rangle$, with modes $|m+(2 n+1),-s\rangle$ for any integer $n$. For example, with $n=0, \Delta E_{m, 1}=E_{0}(2 m+1) \approx E_{0}(2 m)$, while for $n=1$,

$$
\Delta E_{m, 3}=E_{0}(6 m+9) \approx E_{0}(6 m)=3 \Delta E_{m, 1} .
$$

Hence, the corresponding values of $B_{\text {resonance }}$ are in the ratio of 3/1, as noted by Frolov et al. [26].

Figure 6 illustrates the effect of changing the chemical potential $\mu$ or, equivalently, the Fermi velocity $v_{F}=\sqrt{2 \mu / m}$. For square-well confinement, $E_{n}=\hbar^{2} \pi^{2} /\left(2 m W^{2}\right) n^{2}$, where $W$ is the width of the well. For large $n$, the resonance magnetic field is determined by

$$
g \mu_{B} B_{\text {resonance }} \propto 2 n \propto v_{F} .
$$

The locations of the minima in Fig. 6 are roughly proportional to $v_{F}$, as expected from the argument here. In the righthand panel of Fig. 6, we multiply each curve in the left-hand panel by a function found by fitting the data for $B_{x}^{\text {ext }}$ found in Fig. 1(c) of Ref. [26]. In their experiment, the rate of spin
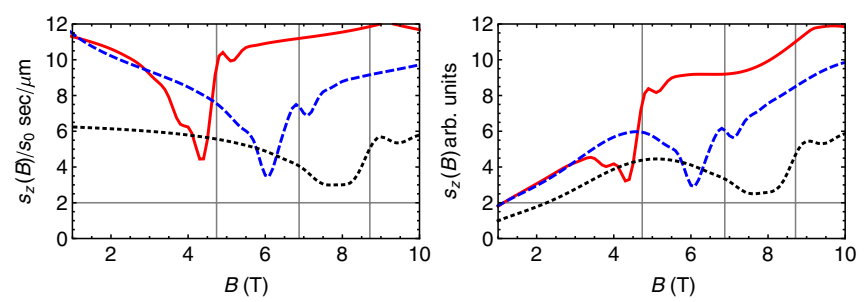

FIG. 6. Spin polarization for three Fermi velocities: solid red line, $v_{F}=0.7 \times 10^{5} \mathrm{~cm} / \mathrm{s}$; dashed blue line, $v_{F}=1.0 \times 10^{5} \mathrm{~cm} / \mathrm{s}$; dotted black line, $v_{F}=1.3 \times 10^{5} \mathrm{~cm} / \mathrm{s}$. Channel width is $1.2 \mu \mathrm{m}$. 
injection $s_{0}$ depends on the magnetic field. This is proportional to the data $s_{x}$ when the external field is in the $x$ direction, since in this case precession should not play a role in the observed spin $(x)$ density. If we assume that $s_{0}(B)$ is in fact independent of the direction of $B$, then we can use $s_{0}(B)$ to model spin- $z$ injection. The curves in the right-hand panel of Fig. 6 are qualitatively similar to the curves in Fig. 2(b) of Ref. [26].

\section{EFFECT OF DISORDER}

The analysis in the previous section neglected the effect of disorder, which could disturb the spin-orbit entanglement upon which the resonant phenomena depend. To evaluate the role of the disorder in Ref. [26], the typical mean-free path $l$ and disorder-induced level broadening $\hbar / \tau$ should be compared to other length and energy scales in the experiment. At a mobility of $4.5 \times 10^{6} \mathrm{~cm}^{2} / \mathrm{V} \mathrm{s}, l \sim$ $20 \mu \mathrm{m}$ and $\hbar / \tau \sim 0.04 \mathrm{~K}$. The typical SO splitting is an order of magnitude larger, $\delta^{\mathrm{SO}} \sim 0.4 \mathrm{~K}$, with the $\mathrm{SO}$ length $l_{\mathrm{SO}}=2 \pi \hbar v_{F} / \delta^{\mathrm{SO}} \sim 11.3 \mu \mathrm{m}$. This definition of $l_{\mathrm{SO}}$ is consistent with Eqs. (39) and (41). The subband separation is $\Delta E \sim 1.5 \mathrm{~K}$, and thus, the separate bands are well resolved $(\Delta E \gg \hbar / \tau)$. $\left\{l_{\mathrm{SO}}, W\right\} \ll l$, so the disorder broadening is the smallest energy scale in the problem and should not play a significant role in Ref. [26]. The remaining length scale, the source-to-drain separation, is comparable to $l$, and the diffusive nature of transport may lead to quantitative (but not qualitative) changes, as we argue below.

The basic physical picture described by the two-subband model of Sec. II also applies to the case of a disordered channel. The injected spin can be thought of as having conserved and nonconserved (precessing) parts, as in Fig. 3. The conserved part diffuses along the channel rather than propagates ballistically at long distances. If the source-to-drain separation $\left|x_{s}-x_{d}\right| \gg l$, the steady-state spin polarization scales with the separation between the source and the drain located at $x=x_{s}$ and $x=x_{d}$; see Fig. 1. It can be explicitly obtained either by Eq. (23) or by the direct solution of the diffusion equation with both source and drain. Both methods are shown in the Appendix to produce the same steady-state polarization profile. The physical picture of the diffusion of the conserved polarization is supported by a microscopic analysis in Secs. IV A and IV B.

These arguments apply if the disorder conserves not just the spin, but also the pseudospin of a state. The condition for preserving the pseudospin is that the disorder potential does not mix the states $|n, \gamma\rangle$ defined in Eq. (28) with opposite $\gamma$ 's. We show below that this condition is satisfied for a generic scattering potential.

The nonconserved part of an injected polarization oscillates on a scale $\lesssim l_{\text {So }}$ away from the source (see Fig. 5, where the oscillation scale is about $5 \mu \mathrm{m}$ ). Weak disorder with $\hbar / \tau \ll \delta^{\mathrm{SO}}$ is ineffective at experimentally relevant distances from the source, $\left|x-x_{s}\right| \lesssim l$. Therefore, the oscillations survive this weak disorder. The conserved part of polarization can even be enhanced by weak disorder, provided $\left|x_{s}-x_{d}\right| \gtrsim l$. In Ref. [26], $\left|x_{s}-x_{d}\right| \simeq l$, and this enhancement is not pronounced. However, making the injector-to-detector separation larger may increase the magnitude of the disorder-driven enhancement of the conserved part of the polarization.

If either the detector is placed close to the node of spin oscillations shown in Figs. 5(c) and 5(d) or the oscillations are smeared out due to the finite size of the injector and detector, the measured signal is determined by the conserved part of the injected spin. Thus, beyond the distance $l_{\text {SO }}$ from the source, the $B$ dependence of a detected spin polarization is chiefly determined by the depolarization factor $\left(\cos ^{2} \theta\right)$ of Eq. (37). Neglecting the variation of $\theta$ over bands,

$$
s_{z}(x, B) \propto \cos ^{2} \theta \approx 1-\frac{\left(\delta^{\mathrm{SO}}\right)^{2}}{\left(E_{z}-\hbar \omega\right)^{2}+\left(\delta^{\mathrm{SO}}\right)^{2}} .
$$

The spin polarization above drops to zero when $B-B_{\text {res }}$ vanishes, with the spin polarization following a Lorentzian curve with a scale of variation of $\delta^{\mathrm{SO}} \sim 0.4 \mathrm{~K}$, which translates into a width of the resonance in magnetic field $\sim 2.3 \mathrm{~T}$. The experimental data confirm this estimate.

We support these general statements with a microscopic analysis for the case of strong disorder, $l \ll l^{\mathrm{SO}}$, in Sec. IV A and the case of weak disorder, $l \gg l^{\mathrm{SO}}$, in Sec. IV B. In both cases, the polarization follows Eq. (43) at distances exceeding $\min \left\{l_{\mathrm{SO}}, \sqrt{l_{\mathrm{SO}} l}\right\}$, at which point the nonconserved part of polarization drops. For $l \gg l^{\mathrm{SO}}$, the polarization decay is ballisticlike, and for $l \ll l^{\mathrm{SO}}$, the polarization follows Hanle-like relaxation [31-33].

\section{A. Spin polarization in the presence of strong disorder, $W \ll l \ll l_{\text {SO }}$}

In this section, we derive expressions for spin-correlation functions in the presence of the disorder with the mean-free path larger than the channel width but smaller than the SO length. We then use these correlation functions to obtain the result Eq. (43). The quasiclassical approximation is justified provided $k_{F} W, k_{F} l \gg 1$. The former condition is equivalent to having many propagating modes. In addition, we assume that the individual modes are well resolved, $W \ll l$.

Since the details of the disorder potential $V_{\mathrm{imp}}(\boldsymbol{r})$ are not essential, we assume it to be Gaussian, with the simplest form of the correlation function,

$$
\left\langle V_{\mathrm{imp}}(\boldsymbol{r}) V_{\mathrm{imp}}\left(\boldsymbol{r}^{\prime}\right)\right\rangle=V\left(z_{1}\right) V\left(z_{2}\right) \delta\left(x-x^{\prime}\right) .
$$

Finally, we simplify the analysis by neglecting the dispersion in angles $\theta_{n}$ of the effective magnetic field; i.e., we set $\theta_{n} \equiv \theta$. This assumption is strictly satisfied at 


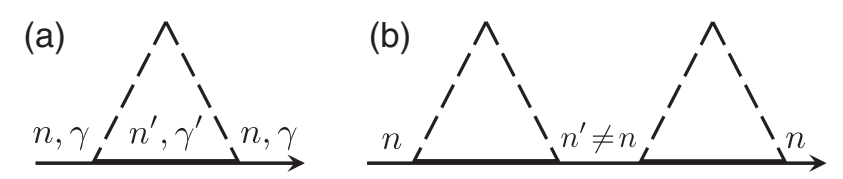

(c)

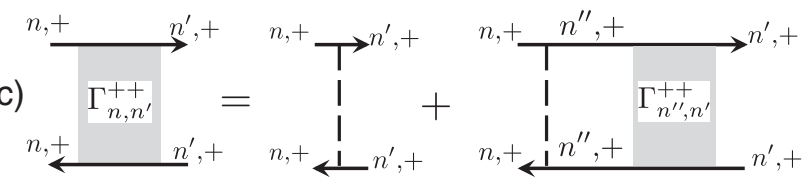

(d)

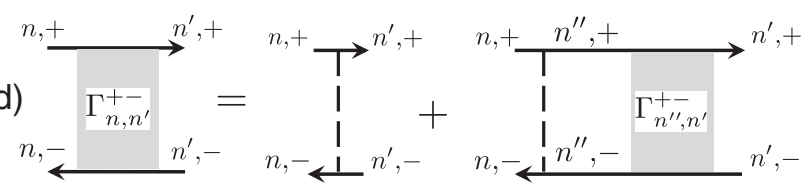

FIG. 7. The diagrammatic representation of (a) the selfconsistent self-energy, Eq. (47), (b) an example of a subleading in $(\Delta E \tau)^{-1}$ contribution. (c),(d) The Dyson equation, Eq. (53), satisfied by a scattering amplitude $\Gamma_{n, n^{\prime}}^{++}$and $\Gamma_{n, n^{\prime}}^{+-}$, respectively. Solid arrowed lines denote Green's functions. The dashed lines stand for disorder averaged scattering amplitudes.

the BSR for a parabolic confinement potential, $\theta_{n}=\pi / 2$. It is also satisfied off the resonance when all $\theta_{n} \approx 0$. In general, a weak dispersion of angles $\theta_{n}$ is expected to cause D'yakonov-Perel'-like relaxation.

We start with the consideration of the Green's function in the self-consistent Born approximation; see Fig. 7. At each Fermi point we linearize the dispersion relation and introduce left- and right-moving species, labeled by the index $r= \pm 1$. We are looking for a Green's function in the form

$$
\hat{G}_{n, \gamma ; r}(\epsilon, p)=|n, \gamma\rangle\langle n, \gamma| G_{n, \gamma ; r}(\epsilon, p),
$$

where the ballistic eigenstates $|n, \gamma\rangle$ are introduced in Eq. (28).

$$
G_{n, \gamma ; r}(\epsilon, p)=\frac{1}{\epsilon-r v_{n} p-\gamma \Delta_{n} / 2+i \hbar / 2 \tau_{n}},
$$

where $\Delta_{n}$ is the band splitting, Eq. (40), and $v_{n}$ is the Fermi velocity of the $n$th band. The rate $\tau_{n}^{-1}$ is determined below within the self-consistent Born approximation using the correlation function Eq. (44). The self-consistency condition is presented graphically in Fig. 7(a). In the case of well-resolved bands, $k_{F} l \gg 1$ or, equivalently, $\Delta E \tau / \hbar \gg 1$, Green's function Eq. (46) is diagonal in the band index $n$. Indeed, the self-energy diagram presented in Fig. 7(b) is smaller than the leading contribution shown in Fig. 7(a) by a factor of $\Delta E \tau \gg 1$. This justifies labeling of Green's function by a single band index $n$. We, therefore, retain only contributions to the self-energy shown in Fig. 7(a). The self-consistency condition represented graphically in Fig. 7(a) with the correlation function Eq. (44) reads

$$
\frac{1}{2 \tau_{n}^{\gamma}}=\operatorname{Im} \int \frac{d p^{\prime}}{2 \pi} \sum_{n^{\prime}, \gamma^{\prime}, r^{\prime}}\left|V_{n, n^{\prime}}^{\gamma \gamma^{\prime}}\right|^{2} G_{n^{\prime}, \gamma^{\prime} ; r^{\prime}}\left(i \epsilon_{m}, p+p^{\prime}\right),
$$

where the disorder matrix elements are

$$
V_{n, n^{\prime}}^{\gamma \gamma^{\prime}}=\left\langle n, \gamma|V(z)| n^{\prime} \gamma^{\prime}\right\rangle
$$

With the explicit expressions for the ballistic eigenstates of the main text and using the fact that the potential does not flip the spin, we write

$V_{n, n^{\prime}}^{++}=\sin \frac{\theta_{n}}{2} \sin \frac{\theta_{n^{\prime}}}{2} V_{n+1, n^{\prime}+1}+\cos \frac{\theta_{n}}{2} \cos \frac{\theta_{n^{\prime}}}{2} V_{n, n^{\prime}}$,

$V_{n, n^{\prime}}^{--}=\cos \frac{\theta_{n}}{2} \cos \frac{\theta_{n^{\prime}}}{2} V_{n+1, n^{\prime}+1}+\sin \frac{\theta_{n}}{2} \sin \frac{\theta_{n^{\prime}}}{2} V_{n, n^{\prime}}$,

$V_{n, n^{\prime}}^{+-}=-i \sin \frac{\theta_{n}}{2} \cos \frac{\theta_{n^{\prime}}}{2} V_{n+1, n^{\prime}+1}+i \cos \frac{\theta_{n}}{2} \sin \frac{\theta_{n^{\prime}}}{2} V_{n, n^{\prime}}$,

$V_{n, n^{\prime}}^{-+}=i \cos \frac{\theta_{n}}{2} \sin \frac{\theta_{n^{\prime}}}{2} V_{n+1, n^{\prime}+1}-i \sin \frac{\theta_{n}}{2} \cos \frac{\theta_{n^{\prime}}}{2} V_{n, n^{\prime}}$,

where

$$
V_{n, n^{\prime}}=\int d z \phi_{n}(z) V(z) \phi_{n^{\prime}}(z)
$$

Because of the large number of propagating modes, $V_{n+1, n^{\prime}+1} \approx V_{n, n^{\prime}}$. And since we assumed $\theta_{n} \approx \theta$, Eq. (49) simplifies to

$$
V_{n, n^{\prime}}^{++}=V_{n, n^{\prime}}^{--}=V_{n, n^{\prime}}, \quad V_{n, n^{\prime}}^{+-}=V_{n, n^{\prime}}^{+-}=0 .
$$

With Eq. (51), evaluation of Eq. (47) is straightforward, with the result

$$
\frac{\hbar}{2 \tau_{n}}=\sum_{n^{\prime}} \frac{V_{n, n^{\prime}}^{2}}{\hbar v_{n^{\prime}}}
$$

where the sum runs over the propagating modes. One can check that the self-energy is diagonal in the band index. This follows from the cancellation of $\gamma^{\prime}= \pm 1$ contributions for the of-diagonal part of the self-energy matrix. Therefore, the assumption of self-energy being diagonal in both $n$ and $\gamma$ indices is shown to be self-consistent. This situation is very similar to the case of a constant Zeeman field, where the form of Eq. (46) is known to hold.

Next, we consider collective density dynamics by analyzing the two-particle correlation functions. Similar to the theory of disordered Fermi liquids [34], the spincorrelation function can be decomposed as a sum of a static and a dynamic part, $\chi=\chi^{s}+\chi^{d}$. The static part $\chi^{s}$ contains contributions to the particle-hole ladder diagrams, Fig. 7, in which the upper and lower Green's functions in Figs. 7(c) and 7(d) are either both retarded or both advanced. The $\chi^{s}$ is 
insensitive to disorder and other energy scales and reduces to a density of states at the Fermi energy, $\chi_{s}(q, \omega) \approx$ $\nu=2 \sum_{n}\left(\hbar \pi v_{n}\right)^{-1}$. The dynamic part $\chi^{d}$ consists of the contributions with upper (lower) Green's functions of a ladder in Figs. 7(c) and 7(d) being retarded (advanced).

We now turn to the analysis of the related retardedadvanced scattering amplitude. Because of Eq. (51), two separate scattering amplitudes can be introduced, $\Gamma^{ \pm \pm}(q, \omega)$ and $\Gamma^{ \pm, \mp}(q, \omega)$, describing the diffusion of the conserved and precessing spin components, respectively. These amplitudes satisfy the corresponding Dyson equations presented graphically in Figs. 7(c) and 7(d),

$$
\Gamma_{n, n^{\prime}}^{\gamma \gamma^{\prime}}=V_{n, n^{\prime}}^{2}+\sum_{n^{\prime \prime}} V_{n, n^{\prime \prime}}^{2} \Pi_{n^{\prime \prime}}^{\gamma \gamma^{\prime}} \Gamma_{n^{\prime \prime}, n}^{\gamma \gamma^{\prime}}
$$

where the polarization operator

$$
\Pi_{n}^{\gamma \gamma^{\prime}}(q, \omega)=\sum_{r= \pm} \int \frac{d p}{2 \pi} G_{n, \gamma ; r}^{R}(\epsilon+\omega, p+q) G_{n, \gamma^{\prime} ; r}^{A}(\epsilon, p)
$$

is easily evaluated with Eq. (46),

$$
\begin{aligned}
\Pi_{n}^{\gamma \gamma^{\prime}}(q, \omega)= & \sum_{r= \pm} \frac{\tau_{n}}{v_{n}}\left[1-i \omega \tau_{n}+i r v_{n} q \tau_{n}\right. \\
& \left.+\frac{i}{2}\left(\gamma-\gamma^{\prime}\right) \Delta_{n} \tau_{n}\right]^{-1} .
\end{aligned}
$$

We first address the dynamics of the component parallel to the effective field by setting $\gamma=\gamma^{\prime}$. At long distances, the spin density is dominated by the spin-diffusion mode away from the source. To identify this mode, we adopt the approach of Ref. [35] and introduce the spectral decomposition of the scattering amplitude as follows:

$$
\sum_{n^{\prime}} V_{n, n^{\prime}}^{2} \Pi_{n^{\prime}}^{ \pm \pm}(0,0) \varphi_{n^{\prime}}^{l}=\lambda_{l} \varphi_{n}^{l}
$$

Here the index $l=0,1, \ldots$ labels eigenfunctions $\varphi_{n}^{l}$ normalized by the condition

$$
\sum_{n} \varphi_{n}^{l} \Pi_{n}^{ \pm \pm}(0,0) \varphi_{n}^{l}=\delta_{l, l^{\prime}}
$$

Substituting the expansion $V_{n, n^{\prime}}^{2}=\sum_{l} a_{n}^{l} \varphi_{n^{\prime}}^{l}$ into Eq. (56) and using Eq. (57), we obtain

$$
V_{n, n^{\prime}}^{2}=\sum_{l} \lambda_{l} \varphi_{n}^{l} \varphi_{n^{\prime}}^{l}
$$

The amplitude in Eq. (53) can be decomposed using Eqs. (56)-(58) as follows:

$$
\Gamma_{n, n^{\prime}}^{ \pm \pm}=\sum_{l} \frac{\lambda_{l}}{1-\lambda_{l}} \varphi_{n}^{l} \varphi_{n^{\prime}}^{l}
$$

The hydrodynamic mode is identified as the one having the eigenvalue $\lambda_{l=0} \approx 1$. Following Ref. [35], this mode is written as

$$
\varphi_{n}^{l=0}=\frac{Z}{2 \tau_{n}}, \quad Z=\sqrt{2}\left(\sum_{n} v_{n}^{-1} \tau_{n}^{-1}\right)^{-1 / 2},
$$

where $Z$ is the normalization factor fixed by the condition Eq. (57). Indeed, because it follows from Eq. (55) that $\Pi^{ \pm \pm}(0,0)=2 \tau_{n} / v_{n}$, and because of Eq. (52), the definition Eq. (56) gives

$$
\sum_{n^{\prime}} V_{n, n^{\prime}}^{2} \Pi_{n^{\prime}}^{ \pm \pm}(0,0) \varphi_{n^{\prime}}^{l=0}=\varphi_{n}^{l=0}
$$

Equation (61) shows that $\varphi^{l=0}$ corresponds to the hydrodynamic pole of the scattering amplitude, Eq. (59), at $q=0$ and $\omega=0$. This pole is a consequence of the conservation of the spin polarization parallel to the effective field. To obtain the dynamics in the long-wavelength, i.e., hydrodynamic, limit, we analyze the small $q$ and small $\omega$ dependence of the $\lambda^{l=0}(q, \omega)$ eigenvalue. At nonzero $q$ and $\omega$, the eigenvalue equation is

$$
\sum_{n^{\prime}} V_{n, n^{\prime}}^{2} \Pi_{n^{\prime}}^{ \pm \pm}(q, \omega) \varphi_{n^{\prime}}^{l=0}=\lambda^{l=0}(q, \omega) \varphi_{n}^{l=0} .
$$

In the hydrodynamic regime, $\omega \tau_{n}, \quad v_{n} q \tau_{n} \ll 1$, the difference

$$
\Pi_{n}^{ \pm \pm}(q, \omega)-\Pi_{n}^{ \pm \pm}(0,0) \approx \frac{2 \tau_{n}}{v_{n}}\left(i \omega \tau_{n}+v_{n}^{2} q^{2} \tau_{n}^{2}\right)
$$

can be considered as a small perturbation of an original eigenvalue problem at $q=0, \omega=0$ with the unperturbed solution Eq. (60). The corresponding perturbation theory has been worked out in Ref. [35], and here we quote the result,

$$
\lambda^{l=0}(q, \omega) \approx 1+i \omega\left\langle\tau^{-1}\right\rangle^{-1}-\langle D\rangle q^{2}\left\langle\tau^{-1}\right\rangle^{-1},
$$

where the averaging over modes is defined as a sum weighed by the density of states,

$$
\langle A\rangle=\frac{\sum_{n} v_{n}^{-1} A_{n}}{\sum_{n} v_{n}^{-1}}
$$

and $D_{n}=v_{n}^{2} \tau_{n}$. In the quasiclassical regime, the number of modes is large, and the sum in Eq. (65) can be replaced by the integral, 


$$
\langle A(n)\rangle_{n} \approx \frac{\int_{-k_{F}}^{k_{F}} d k A(k)\left(k_{F}-k^{2}\right)^{-1 / 2}}{\int_{-k_{F}}^{k_{F}} d k\left(k_{F}-k^{2}\right)^{-1 / 2}},
$$

where $k_{F}$ is the bulk Fermi momentum. Equation (66), in turn, corresponds to an angular integration over the Fermi surface with the energy-independent density of states. In the same limit, the relaxation rate $\langle\tau\rangle^{-1}$ and the diffusion coefficient $\langle D\rangle$ approach their corresponding bulk values $1 / \tau$ and $k_{F}^{2} / m^{2}(\tau / 2)$ for a two-dimensional electron gas in the presence of $\delta$-correlated disorder. In the case of the diffusion coefficient, the numerical prefactor appears as a result of the identity

$$
\frac{\int_{-1}^{1} d x x^{2}\left(1-x^{2}\right)^{-1 / 2}}{\int_{-1}^{1} d x\left(1-x^{2}\right)^{-1 / 2}}=1 / 2 .
$$

Keeping only the hydrodynamic mode in the expansion, Eq. (59), as appropriate at large distances from the spin source, we finally obtain

$$
\Gamma_{n, n^{\prime}}^{ \pm \pm} \approx \frac{\varphi_{n}^{l=0} \varphi_{n^{\prime}}^{l=0}\left\langle\tau^{-1}\right\rangle}{i \omega-\langle D\rangle q^{2}}
$$

which is natural for diffusion without spin-precession and spin-flip processes.

The diffusion of the nonconserved component of the spin polarization is accompanied by oscillation. This manifests itself in polarization operators at small $q$ and $\omega$,

$$
\Pi_{n}^{ \pm \mp}(q, \omega)-\Pi_{n}^{ \pm \mp}(0,0) \approx \frac{2 \tau_{n}}{v_{n}}\left(i \omega \tau_{n}+v_{n}^{2} q^{2} \tau_{n}^{2} \mp i \Delta_{n} \tau_{n}\right),
$$

which differs from Eq. (63) by the presence of a spinsplitting contribution. Similarly to Eq. (67), we obtain

$$
\Gamma_{n, n^{\prime}}^{ \pm \mp} \approx \frac{\varphi_{n}^{l=0} \varphi_{n^{\prime}}^{l=0}\left\langle\tau^{-1}\right\rangle}{i \omega-\langle D\rangle q^{2} \mp i\langle\Delta\rangle} .
$$

The most singular contributions to a dynamical spin susceptibility are

$$
\begin{aligned}
\chi^{d}(q, \omega) \approx & \frac{i \omega}{2 \pi} \sum_{\gamma= \pm} \sum_{n, n^{\prime}}\left[\cos ^{2} \theta \Pi_{n}^{\gamma, \gamma} \Gamma_{n, n^{\prime}}^{\gamma, \gamma} \Pi_{n^{\prime}}^{\gamma, \gamma}\right. \\
& \left.+\sin ^{2} \theta \Pi_{n}^{\gamma,-\gamma} \Gamma_{n, n^{\prime}}^{\gamma,-\gamma} \Pi_{n^{\prime}}^{\gamma,-\gamma}\right]
\end{aligned}
$$

where the $\cos \theta$ and $\sin \theta$ are the matrix elements of the spin operator in the $|n, \pm\rangle$ basis introduced in the discussion of the ballistic case. In Eq. (70), the polarization operators can be evaluated at $q=0$ and $\omega=0$, so that from Eq. (55), $\Pi_{n}^{\gamma \gamma^{\prime}} \approx 2 \tau_{n} / v_{n}$. Then substitution of Eqs. (60), (67), and (69) into Eq. (70) gives

$$
\begin{aligned}
& \chi^{d}(q, \omega) \approx \nu \cos ^{2} \theta \frac{\omega}{\omega+i\langle D\rangle q^{2}} \\
& \quad+\frac{\nu}{2} \sin ^{2} \theta\left(\frac{\omega}{\omega-\langle\Delta\rangle+i\langle D\rangle q^{2}}+\frac{\omega}{\omega+\langle\Delta\rangle+i\langle D\rangle q^{2}}\right) .
\end{aligned}
$$

The first term in this equation describes diffusion of the spin component parallel to the effective field. The second term describes the diffusion of oscillating spin polarization (coming from precessing pseudospins). The stationary spin polarization obtained by a substitution of Eq. (71) in Eq. (23) reads

$$
\begin{aligned}
s_{z}(x) \approx & s_{0} \cos ^{2} \theta\left(\left|x_{s}-x_{d}\right| / 2\langle D\rangle\right) \\
& -\frac{s_{0} l_{H}}{2^{3 / 2}\langle D\rangle} \sin ^{2} \theta e^{-\left|x-x_{s}\right| / l_{H}} \cos \left(\frac{\pi}{4}+\frac{\left|x-x_{s}\right|}{l_{H}}\right) \\
& +\frac{s_{0} l_{H}}{2^{3 / 2}\langle D\rangle} \sin ^{2} \theta e^{-\left|x-x_{d}\right| / l_{H}} \cos \left(\frac{\pi}{4}+\frac{\left|x-x_{d}\right|}{l_{H}}\right),
\end{aligned}
$$

where the Hanle length $l_{H}=\sqrt{2 \hbar\langle D\rangle /\langle\Delta\rangle}=\sqrt{2 l_{\mathrm{SO}} l} \gg l$. For the particular geometry, $x_{s}<x_{d}<x$ with $\left|x_{s}-x_{d}\right| \gg$ $l_{H} \gg l$ and $x$ within a few $l_{H}$ of $x_{s}$, the last term due to the drain in Eq. (72) can be omitted. At $x-x_{s} \gg l_{H}$, the second term due to the source in Eq. (72) is negligible as well and the result Eq. (43) is obtained using Eqs. (37) and (38) and replacing $\delta_{n}^{\mathrm{SO}} \approx \delta^{\mathrm{SO}}$.

In the above derivation, the dispersion in $\theta_{n}$ angles has been neglected. We expect the variation of $\theta_{n}$ to induce randomness in the effective field and, therefore, cause spin relaxation akin to a D'yakonov-Perel' mechanism of spin relaxation. Nevertheless, the corresponding relaxation rate is expected to be suppressed relative to its bulk value because such band-to-band variations of $\theta_{n}$ are rather small. In the parabolic confining potential at the resonance, $\theta_{n}=\pi / 2$ for all $n$. Likewise, off the resonance, $\theta_{n} \approx 0$. This spin relaxation is left for future studies.

\section{B. Spin polarization in the presence of weak disorder, $W \ll l_{\text {SO }} \ll l$}

Weak disorder has little effect on the nonconserved (precessing) part of spin polarization, whereas the conserved part still diffuses along the channel for distances exceeding the mean-free path. Therefore, the second term of Eq. (41), which is valid in the ballistic case, applies for the nonconserved component, and the first term of Eq. (72) is appropriate for the conserved part, which is still determined by a simple diffusion equation. For distances much more than $l_{\text {SO }}$, away from the source the signal is dominated by the conserved part and the BSR effect is qualitatively described by Eq. (43).

To justify this, we begin with Eq. (54), which remains valid. The components of the polarization operator 
$\Pi_{n}^{\gamma \gamma^{\prime}}(q, \omega)$ with $\gamma \neq \gamma^{\prime}$ contain the factor $\Delta_{n} \tau_{n}$ in the denominator, where $\Delta_{n} \tau_{n} \gg 1$ [Eq. (55)]:

$$
\Pi_{n}^{ \pm \mp}(q, \omega)=\sum_{r= \pm} \frac{\tau_{n}}{v_{n}}\left[1-i \omega \tau_{n}+i r v_{n} q \tau_{n} \pm i \Delta_{n} \tau_{n}\right]^{-1} .
$$

In the interval $x \gg l_{\mathrm{SO}}$, we can approximate Eq. (73) as

$$
\Pi_{n}^{ \pm \mp}(q, \omega) \simeq \pm 2 i \frac{\tau_{n}}{v_{n}}\left(\Delta_{n} \tau_{n}\right)^{-1}
$$

From Eq. (52), we can estimate the scattering vertex $V^{2} \simeq \hbar v / \tau$, where the subband indices are omitted for clarity. This yields an estimate of the product

$$
V^{2} \Pi^{ \pm \mp} \simeq \Delta \tau \ll 1
$$

It follows from Eq. (75) that the components $\Pi^{ \pm \mp}$ are approximately their ballistic counterparts, as the Dyson series [Fig. 7(d)] produces corrections to the ballistic result that are small by a factor $\sim(\Delta \tau / \hbar)^{-1}=l_{\mathrm{sO}} / l$.

The components $\Pi^{ \pm \pm}$, Eq. (55) with $\gamma=\gamma^{\prime}= \pm$, do not contain the parameter $\Delta \tau$, so

$$
\Pi_{n}^{ \pm \pm}(q, \omega)=\sum_{r= \pm} \frac{\tau_{n}}{v_{n}}\left[1-i \omega \tau_{n}+i r v_{n} q \tau_{n}\right]^{-1},
$$

which describes the diffusion of the conserved part regardless of the ratio $l / l_{\mathrm{SO}}$. Combining these observations, for the steady-state spin polarization we obtain

$$
\begin{aligned}
s_{z}(x, B)= & s_{0} \cos ^{2} \theta \frac{\left|x_{s}-x_{d}\right|}{2\langle D\rangle} \\
& +\frac{s_{0}}{2 \sum_{n} v_{n}^{-1}} \sum_{n} v_{n}^{-2} \sin ^{2} \theta_{n} \cos \left[\frac{2 \pi}{l_{n}}\left(x-x_{d}\right)\right] .
\end{aligned}
$$

In Eq. (77), the source contribution was omitted, as we assume the source is located far enough (many $l_{H}$ ) from the point of observation $x$.

\section{Comparison to the case of no Zeeman splitting, $E_{Z}=0$}

We now comment on the relation of the present analysis to previous studies of spin dynamics in narrow channels in the diffusive regime. The spin dynamics in the limit $E_{Z}=0$ and with the mean-free path the shortest length scale in the problem was studied in Refs. [17,22]. The dynamics in the spin channel was described by the spin-1 spinor $\psi$ with the components being linear combinations of spin polarization. The collective modes in this regime were identified as solutions of a $3 \times 3$ matrix equation [17,36], which we reproduce here for the sake of comparison to the present problem, $\left[D\left(q_{x}-2 m \alpha_{+} \hat{J}_{z}\right)^{2}+D\left(-i \partial_{z}-2 m \alpha_{-} \hat{J}_{x}\right)^{2}\right] \psi_{q}=$ $-i \omega \psi_{q}$, where $\omega$ is the frequency of a collective mode.
The matrices $\hat{J}_{i}, i=1,2,3$, represent spin-1 angular momentum.

In the absence of Zeeman splitting, the term proportional to $\alpha_{-}$can be eliminated by a suitable gauge transformation [17]. If the channel's width $W$ is smaller than $\left(m \alpha_{-}\right)^{-1}$, this transformation is close to identity and, therefore, has a small influence on the $\alpha_{+}$term that can be treated by perturbation theory. This is true even if the mean-free path is larger than the channel width and $W \ll\left(m \alpha_{-}\right)^{-1}$. The reason is that the transverse subbands' energy separation exceeds the typical matrix element of $\alpha_{-} p_{z} \sigma_{x}$ in this regime. In other words, the $\alpha_{-}$term is largely irrelevant and the spin dynamics is controlled by the $\alpha_{+}$term alone. The modes are then classified as eigenstates of the $\hat{J}_{z}$ operator. The slowest modes include the so-called spinspiral configurations [22].

We now compare this situation to the spin dynamics studied in this paper at the BSR. At $E_{z} \approx \hbar \omega_{c}$, it is $\alpha_{-}$that is more important and $\alpha_{+}$less relevant. This is due to the strong mixing of subbands $|n+1, \downarrow\rangle$ and $|n, \uparrow\rangle$, as discussed in Sec. II. We emphasize that the $\alpha_{-}$term cannot be gauged out close to the BSR because it strongly mixes the above subbands, which are near degeneracy at the resonance. Instead, the pairs $|n+1, \downarrow\rangle$ and $|n, \uparrow\rangle$ entangled by the $\alpha_{-}$term define the pseudospin space for each $n$. In the above discussion, these states were labeled by $|n, \gamma\rangle$, with $\gamma= \pm 1$. To derive expressions that can be compared with previous studies, we introduce the spin-1 spinor $\psi^{(n)}$ describing the total angular momentum of electron-hole pairs, [36] in a pseudospin subspace $|n, \gamma\rangle, \gamma= \pm 1$. For the purposes of this work, it is sufficient to consider $E_{Z}=\hbar \omega_{c}$.

The equation for collective modes obtained above [(Eq. (62)] can be rewritten in a form similar to that used in Ref. [17] as follows:

$$
\begin{aligned}
& \sum_{n^{\prime}} V_{n n^{\prime}}^{2} \frac{2 \tau_{n^{\prime}}}{v_{n^{\prime}}}\left[1+i \omega \tau_{n^{\prime}}+D_{n^{\prime}}\left(q_{x}-2 \alpha_{+} \hat{J}_{z}\right)^{2} \tau_{n^{\prime}}\right. \\
& \left.+\delta_{n^{\prime}}^{\mathrm{SO}} \hat{J}_{x}\right] \psi^{\left(n^{\prime}\right)}=\lambda(q, \omega) \psi^{(n)},
\end{aligned}
$$

where the splitting $\delta_{n}^{\text {SO }}$ is solely due to the $\alpha_{-}$term as defined by Eq. (39). The diffusion pole is $[1-\lambda(q, \omega)]^{-1}$, where $\lambda(q, \omega)$ is the lowest hydrodynamic eigenfunction. For $\alpha_{+}=0$, Eq. (78) simplifies. The collective modes are labeled by eigenstates of $\hat{J}_{x}$, and the results of Sec. IV A are recovered. The conserved polarization diffusion pole, Eq. (67), is obtained for eigenstates with $\hat{J}_{x} \psi^{(n)}=0$. The precessing polarization Hanle-like pole, Eq. (69), is obtained for $\widehat{J}_{x} \psi^{(n)}= \pm \psi^{(n)}$.

The inclusion of the $\alpha_{-}$term, in general, makes the above classification inapplicable. We can estimate the importance of this term by comparing its typical value and $\delta_{-} \sim \alpha_{-} p_{F}$. At typical wave vectors $q \simeq \sqrt{\alpha_{-} \Delta / D}$, the ratio of the two terms is $\sim\left(\alpha_{+} / \alpha_{-}\right) \sqrt{\alpha_{-} p_{F} \tau}$. In the diffusive regime studied in Refs. [17,22], $\alpha_{-} p_{F} \tau \ll 1$, and, therefore, the $\alpha_{+}$term is expected to produce little change in the spin dynamics 
within each pseudospin space. In the presence of intersubband scattering, it leads to D'yakonov-Perel'-like relaxation, and is expected to affect the shape of the resonance. Nevertheless, the general trend of the nonequilibrium spin polarization to form a dip at the resonance remains intact.

\section{CONCLUDING REMARKS}

We study theoretically the phenomenon of BSR in quasione-dimensional channels. Our theory reliably reproduces the drop in the nonlocal voltage (which presumably reflects local spin density) induced by nonequilibrium spin polarization in both ballistic and diffusive regimes. When the Zeeman splitting is comparable to the energy scale of transverse quantization, the bands become doubly degenerate, as in Fig. 2. By hybridizing these pairs of bands, SO coupling causes the precession of the injected spin polarization, as in Fig. 3. In the ballistic regime, the variation of the injected spin density is due to the spatial beating of various modes in the channel. The magnitude of the oscillation is reduced by increasing the size of the source. In the diffusive regime, the dynamics of injected spins is a Hanle-like relaxation.

In this paper, we model the spin injection as a continuous influx of a quasiequilibrium polarization, as in Ref. [37]. While such an approximation may apply to spin injection from a ferromagnetic contact to a bulk semiconductor [38], its application to injection via a QPC has yet to be studied. Experimentally, the voltage is fixed, not the spin injection rate. A more rigorous way of describing the injection would be to use a Landauer approach. In a multiterminal geometry, this requires solution of a quantum-mechanical scattering problem for electrons injected via the QPC.

Although we have captured the gross features of BSR, a more accurate modeling of a spin injection is necessary for a detailed description of spin transport in channels contacted by a QPC. For example, when a band is just touching the Fermi level, the velocity of the carriers along the channel is nearly zero, so that if the injection rate is kept constant, a divergent spin accumulation would result. The injection rate should instead become zero due to perfect reflection at the QPC, which eliminates the divergence. We also note that the effective size of an injection or detection region depends on the microscopic details of a QPC. Within our theory, therefore, this size should be regarded as a phenomenological parameter. In Fig. 5, the injector size is taken to be $0.5 \mu \mathrm{m}$. If one takes $2 \mu \mathrm{m}$ instead, the spin polarization is noticeably smoothed out, as seen in Fig. 8.

Oscillations in the nonlocal voltage due to ballistic spin precession have been reported in Ref. [39]. The oscillations seen in our Fig. 5 reflect this observation. Injection via the QPC differs from injection from the long contact bar used in Ref. [39] and better resembles Fig. 8, with an effectively larger injector and detector region. A more detailed study of the spin injection across a QPC will be helpful to develop

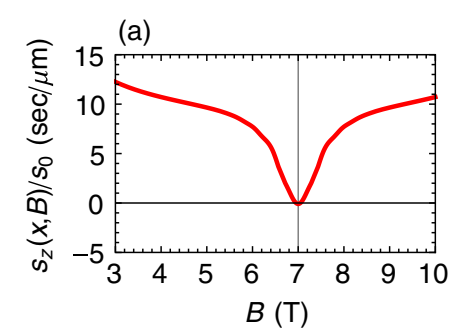

(b)
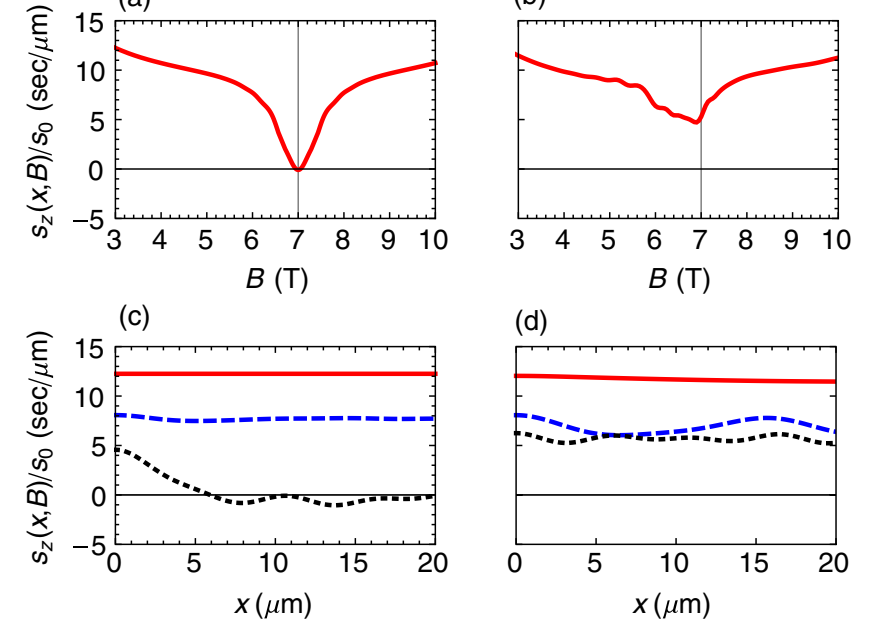

(d)

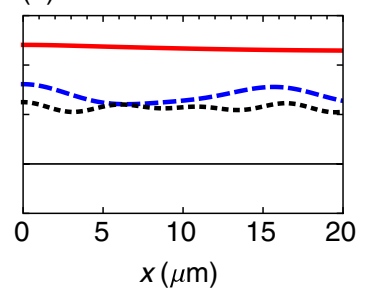

FIG. 8. The same as Fig. 5 but with an effective spin injector size of $2 \mu \mathrm{m}, 4$ times larger than $0.5 \mu \mathrm{m}$ used in Fig. 5. In this case, the effective injector size is comparable to the typical channel width $W=1.2 \mu \mathrm{m}$. The dip in spin polarization is more pronounced for parabolic lateral confinement (a) as the resonance condition, Eq. (21), can be satisfied for all bands simultaneously, which is not the case for the infinite square-well confinement (b). This is due to the washing out of an oscillatory precessing polarization component with distance. The suppression of oscillations occurs for larger injector sizes and is present for both the parabolic (c) and square-well (d) confinements.

further understanding, and will be addressed in forthcoming studies.

The BSR phenomenon is an ideal setting to study the role of electron-electron interaction in spin transport and spin relaxation in confined geometries, which was the primary motivation for this work. Of particular interest is an interaction-induced renormalization of the resonant field, which will be studied in the future, based on the theory above. Spin relaxation and coherence of the precessing pseudospins in the channel are likely to yield additional surprises.

\section{ACKNOWLEDGMENTS}

We thank J. A. Folk and A. M. Finkel'stein for useful discussions. M. K. acknowledges the support of the University of Iowa. M.E. F. was supported in part by C-SPIN, one of six centers of STARnet, a Semiconductor Research Corporation program, sponsored by MARCO and DARPA.

Note added.-Recently, we became aware of Ref. [40], which also treats ballistic spin resonance.

\section{APPENDIX: STATIONARY SPIN POLARIZATION}

In this Appendix, we derive the general relation, Eq. (23), assuming linear response, and illustrate it for simple diffusion. For clarity, we consider a steady influx of a specified particle density; the number of particles 
imparted into the system per unit time and per unit volume is the flux $F_{\rho}(\boldsymbol{x})$. In order to relate the steady-state density to the retarded density correlation function $\chi_{\rho}(\boldsymbol{x}, t)$, we rely on the following auxiliary result proven in Ref. [37]. If an excess density $\rho(\boldsymbol{x})$ is prepared at an initial time instant $t_{i}$, the subsequent time evolution of the density at a given time $t$ and location $\boldsymbol{x}, \rho(\boldsymbol{x}, t)$, is determined by the correlation function $\chi_{\rho}(\boldsymbol{x}, t)$ according to

$$
\rho\left(\boldsymbol{x}, t>t_{i}\right)=-i \int \frac{d \omega}{2 \pi} \int d \boldsymbol{x}^{\prime} \int d \boldsymbol{x}^{\prime \prime} e^{-i \omega\left(t-t_{i}\right)} \frac{\left[\chi_{\rho}\left(\boldsymbol{x}-\boldsymbol{x}^{\prime}, \omega\right)-\chi_{\rho, \mathrm{st}}\left(\boldsymbol{x}-\boldsymbol{x}^{\prime}\right)\right]}{\omega-i 0^{+}} \chi_{\rho, \mathrm{st}}^{-1}\left(\boldsymbol{x}^{\prime}-\boldsymbol{x}^{\prime \prime}\right) \rho\left(\boldsymbol{x}^{\prime \prime}, t_{i}\right),
$$

where the static correlation function $\chi_{\rho, \mathrm{st}}\left(\boldsymbol{x}^{\prime}-\boldsymbol{x}^{\prime}\right)=\chi_{\rho}\left(\boldsymbol{x}^{\prime}-\boldsymbol{x}^{\prime}, \omega=0\right)$. During each infinitesimal time interval $\left[t_{i}, t_{i}+d t_{i}\right]$, the density $F_{\rho}(\boldsymbol{x}) d t_{i}$ is imparted into the system. This density is evolving in time according to Eq. (A1). In the linear response regime, the separate contributions of each of the time intervals add up to yield

$$
\rho(\boldsymbol{x}, t)=-i \int_{-\infty}^{t} d t_{i} \int \frac{d \omega}{2 \pi} \int d \boldsymbol{x}^{\prime} \int d \boldsymbol{x}^{\prime \prime} e^{-i \omega\left(t-t_{i}\right)} \frac{\left[\chi_{\rho}\left(\boldsymbol{x}-\boldsymbol{x}^{\prime}, \omega\right)-\chi_{\rho \mathrm{st}}\left(\boldsymbol{x}-\boldsymbol{x}^{\prime}\right)\right]}{\omega-i 0^{+}} \chi_{\rho, \mathrm{st}}^{-1}\left(\boldsymbol{x}^{\prime}-\boldsymbol{x}^{\prime \prime}\right) F_{\rho}\left(\boldsymbol{x}^{\prime \prime}\right) .
$$

For a flux that is turned on adiabatically, $F_{\rho}\left(\boldsymbol{x}^{\prime \prime}\right)$ is replaced in Eq. (A2) by $F_{\rho}\left(\boldsymbol{x}^{\prime \prime}, t_{i}\right)=F_{\rho}\left(\boldsymbol{x}^{\prime \prime}\right) \exp \left(\delta^{+} t_{i}\right)$. The steady-state result is then obtained by taking the limit $\delta \rightarrow 0$ at the end of the calculation. With this regularization, the integration over the injection time $t_{i}$ is straightforward and yields the following expression:

$$
\rho(\boldsymbol{x})=-\int \frac{d \omega}{2 \pi} \int d \boldsymbol{x}^{\prime} \int d \boldsymbol{x}^{\prime \prime} \frac{\left[\chi_{\rho}\left(\boldsymbol{x}-\boldsymbol{x}^{\prime}, \omega\right)-\chi_{\rho, \mathrm{st}}\left(\boldsymbol{x}-\boldsymbol{x}^{\prime}\right)\right]}{\left(\omega-i 0^{+}\right)\left(\omega-i \delta^{+}\right)} \chi_{\rho, \mathrm{st}}^{-1}\left(\boldsymbol{x}^{\prime}-\boldsymbol{x}^{\prime \prime}\right) F_{\rho}\left(\boldsymbol{x}^{\prime \prime}\right) .
$$

The integration over the frequency $\omega$ can be done by closing the contour of integration in the upper half of a complex $\omega$ plane due to the fast decay of the integrand at large $\omega$. It is essential that the retarded correlation function $\chi_{\rho}\left(\boldsymbol{x}-\boldsymbol{x}^{\prime}, \omega\right)$ is analytic in the area enclosed by the above contour. Using the residue theorem and introducing spatial Fourier harmonics, we rewrite Eq. (A3) as

$$
\rho(\boldsymbol{x})=-\left.i \int \frac{d^{d} q}{(2 \pi)^{d}} e^{i \boldsymbol{q} \boldsymbol{x}}\left[\partial_{\omega} \chi_{\rho}(\boldsymbol{q}, \omega) \chi_{\rho, \mathrm{st}}^{-1}(\boldsymbol{q})\right]\right|_{\omega \rightarrow i \delta} F_{\rho}(\boldsymbol{q}),
$$

where $d$ is the dimension of space. We note that the order of the limits taken with the regularizing infinitesimals $0^{+}$and $\delta^{+}$in (A4) is not important.

The arguments leading to Eq. (A4) can be repeated for spin injection. The steady state spin polarization density $\boldsymbol{s}(\boldsymbol{x})$ is determined by the spin polarization rate $\boldsymbol{F}$ following a similar approach to Eq. (A4), yielding

$$
\boldsymbol{s}(\boldsymbol{x})=-i \int \frac{d^{d} q}{(2 \pi)^{d}} e^{i \boldsymbol{q} x}\left[\partial_{\omega} \bar{\chi}(\boldsymbol{q}, \omega) \bar{\chi}^{-1}(\boldsymbol{q})\right] \mid \underset{\omega \rightarrow i \delta}{\boldsymbol{F}_{\boldsymbol{q}}}
$$

where $\bar{\chi}(\boldsymbol{q}, \omega)$ is the dynamical spin polarization tensor and matrix multiplication is assumed.
To realize the specific case treated in this paper, we assume the injection rate $\boldsymbol{F}(\boldsymbol{x})$ to be constant across the channel and introduce a spin density that has been integrated over the channel cross section,

$$
\hat{\boldsymbol{s}}(x)=\frac{1}{2} \int_{-\infty}^{+\infty} d z\left[\psi_{s_{1}}^{\dagger}(x, z) \boldsymbol{\sigma}_{s_{1}, s_{2}} \psi_{s_{2}}(x, z)\right],
$$

where $\boldsymbol{\sigma}$ are the Pauli matrices and $s_{1,2}$ are dummy spin indices. Integrating Eq. (A5) over the cross section of a quasi-one-dimensional channel and using the definition Eq. (A6), for the steady-state value of the integrated spin density we obtain

$$
s(x)=-\left.i \int \frac{d q}{2 \pi} e^{i q x}\left[\partial_{\omega} \chi(q, \omega) \chi^{-1}(q)\right]\right|_{\omega \rightarrow i \delta} \boldsymbol{F}_{q},
$$

where the integrated spin polarization tensor

$$
\chi_{q, \omega}^{i j}=-i \int_{0}^{\infty} d t \int d x e^{-i q x+i \omega t}\left\langle\left[\hat{s}_{i}(x, t), \hat{s}_{j}(0,0)\right]\right\rangle .
$$

We now consider injection of a $z$ component of polarization, which is the experimental geometry, and set $\boldsymbol{F}(\boldsymbol{x})=\hat{z} F(x)$. The resulting $z$ component of the integrated spin polarization from Eqs. (A7) and (A8) is

$$
s_{z}(x)=-\left.i \int \frac{d q}{2 \pi} e^{i q x} \sum_{j=x, y, z}\left\{\partial_{\omega} \chi^{z j}(\boldsymbol{q}, \omega)\left[\chi^{-1}(\boldsymbol{q})\right]^{j z}\right\}\right|_{\omega \rightarrow i \delta} F_{q} .
$$


For the geometry considered in the main text, only $j=z$ provides nonzero contributions to Eq. (A9), for although $\hat{s}_{z}(x)$ has nonzero matrix elements (only within the twodimensional eigenstate subspaces $\alpha|n+1, \uparrow\rangle+\beta|n, \downarrow\rangle$, $\left.\beta^{*}|n+1, \uparrow\rangle-\alpha^{*}|n, \downarrow\rangle\right)$, all four matrix elements in the same subspace of $\hat{s}_{x}(x)$ and $\hat{s}_{y}(x)$ are zero. Therefore, Eq. (A9) reduces to

$$
\left.s_{z}(x)=-i \int \frac{d q}{2 \pi} e^{i q x} \partial_{\omega} \chi^{z z}(q, \omega)\left[\chi^{-1}(q)\right]\right]_{\omega \rightarrow i \delta}^{z z} F_{q} .
$$

Finally, by writing $\chi^{z z}(q, \omega)=\chi(q, \omega)$ and $\chi^{z z}(q)=\chi(q)$, Eq. (23) is obtained.

\section{Steady state in diffusive regime}

To illustrate the result Eq. (A4), we consider diffusion in $d$ spatial dimensions. The density-correlation function reads

$$
\chi_{\rho}(q, \omega)=\nu_{d} \frac{D q^{2}}{D q^{2}-i \omega}
$$

where $\nu_{d}$ is the density of states and $D$ is the diffusion coefficient. Substitution of Eq. (A11) in Eq. (A4) yields

$$
\rho(x)=\left.\int \frac{d^{d} q}{(2 \pi)^{d}} \frac{e^{i q x} F_{\rho}(\boldsymbol{q})}{D q^{2}-i \omega}\right|_{\omega \rightarrow i \delta} .
$$

Consider the localized injection source of an excess density at the origin $\boldsymbol{x}=0$. For the injection rate, we have $F_{\rho}(\boldsymbol{x})=s_{0} \delta(\boldsymbol{x})$, which gives $F_{\rho}(\boldsymbol{q})=s_{0}$. It follows that for the localized source, the integral in Eq. (A12) diverges for $d=1,2$ and converges in higher dimensions. This fact reflects the Polýa recurrence theorem, which states that the random walker will return to its original location in a finite time for $d=1,2$, while in $d>3$, the recurrence time is infinite. For a generic source, the integral in Eq. (A12) diverges. Correspondingly, the jamming in $d=1,2$ prevents the system from reaching the steady state. For $d>3$, the jamming is reduced, and the steady state is reached, as given by the convergent integral [Eq. (A12)]. Note that the convergence at large wave vectors is achieved due to the finite size of any realistic injector.

The steady state is realized in $d=1,2$, provided the total flux injected is zero. Indeed, in this case, $F_{\rho}(\boldsymbol{q}=0)=0$, and the integral in Eq. (A12) is convergent. The simplest realization of this situation is the combination of spatially separated pointlike source and drain. Focusing on $d=1$, we write the injection profile as

$$
F(x)=s_{0}\left[\delta\left(x-x_{s}\right)-\delta\left(x-x_{d}\right)\right]
$$

which describes the source and drain located at $x=x_{s}$ and $x=x_{d}$, respectively. The Fourier image of Eq. (A13) is

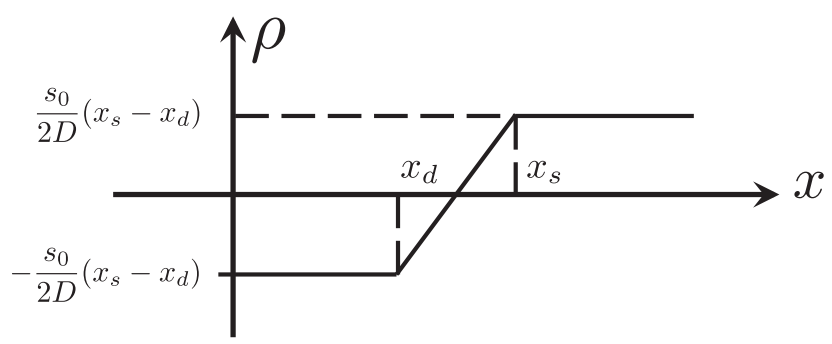

FIG. 9. The steady-state density distribution, Eq. (A15), obtained by the integration in Eq. (A12) with a source and drain located at $x=x_{s}$ and $x=x_{d}$ as represented by the injection rate, Eq. (A13).

$$
F(q)=s_{0}\left[e^{-i q x_{s}}-e^{-i q x_{d}}\right] .
$$

Since the total injected flux $F(q=0)=0$, the steady state becomes possible and the integral in Eq. (A12) becomes convergent. It is important to keep $\delta$ finite in the course of this integration and set $\delta=0$ only at the end of the calculation. This widely used limiting procedure reflects our scheme of adiabatically turning on the sources starting at the remote past. The straightforward integration over $q$ gives the following result:

$$
S(x)=\frac{s_{0}}{2 D}\left[\left|x-x_{d}\right|-\left|x-x_{s}\right|\right] .
$$

The result, Eq. (A15), can also be easily obtained by solving the diffusion equation in the steady state $\partial_{x}^{2} \rho(x)=0$ with the boundary conditions Eq. (A20) derived in the next section. The solution, Eq. (A15), is presented graphically in Fig. 9.

Equation (A12) can also be written in real space,

$$
\rho(x)=\int_{0}^{\infty} d t \int_{-\infty}^{\infty} d x^{\prime} D_{c}\left(x-x^{\prime}, t\right) F_{\rho}\left(x^{\prime}\right)
$$

where the diffusion kernel

$$
D_{c}(x, t)=\frac{\exp \left(-x^{2} / 4 D t\right)}{\sqrt{4 \pi D t}} .
$$

Equations (A16) and (A17) can be generalized easily to include spin precession, drift, and relaxation leading to, e.g., Eq. (1) of Ref. [38].

\section{Boundary conditions for the diffusion in one spatial dimension}

We integrate the diffusion equation with the source

$$
\partial_{t} \rho=D \partial_{x}^{2} \rho+F(x)
$$

over the interval $b_{1}<x<b_{2}$ in the steady state $\partial_{t} \rho=0$ to obtain 


$$
\int_{b_{1}}^{b_{2}} d x F(x)=-D \partial_{x} \rho\left(x=b_{2}\right)+D \partial_{x} \rho\left(x=b_{1}\right) .
$$

We now apply the general Eq. (A19) to Eq. (A13), with $b_{1}=x_{s}-\epsilon,\left(x_{d}-\epsilon\right)$ and $b_{2}=x_{s}+\epsilon\left(x_{d}+\epsilon\right)$, and take the limit $\epsilon \rightarrow 0$. As a result, the following boundary conditions are obtained:

$$
\begin{aligned}
& -D \partial_{x}\left(x=x_{s}+0^{+}\right)+D \partial_{x}\left(x=x_{s}-0^{+}\right)=s_{0}, \\
& -D \partial_{x}\left(x=x_{d}+0^{+}\right)+D \partial_{x}\left(x=x_{d}-0^{+}\right)=-s_{0} .
\end{aligned}
$$

[1] S. A. Wolf, D. D. Awschalom, R. A. Buhrman, J.M. Daughton, S. von Molnár, M. L. Roukes, A. Y. Chtchelkanova, and D. M. Treger, Spintronics: A SpinBased Electronics Vision for the Future, Science 294, 1488 (2001).

[2] D. D. Awschalom and M.E. Flatté, Challenges for Semiconductor Spintronics, Nat. Phys. 3, 153 (2007).

[3] S. Datta and B. Das, Electronic Analog of the Electro-optic Modulator, Appl. Phys. Lett. 56, 665 (1990).

[4] J. Schliemann, J. C. Egues, and D. Loss, Nonballistic SpinField-Effect Transistor, Phys. Rev. Lett. 90, 146801 (2003).

[5] K. Hall and M. E. Flatté, Performance of a Spin-Based Insulated Gate Field Effect Transistor, Appl. Phys. Lett. 88, 162503 (2006).

[6] S. Heedt, C. Morgan, K. Weis, D. E. Bürgler, R. Calarco, H. Hardtdegen, D. Grützmacher, and T. Schäpers, Electrical Spin Injection into InN Semiconductor Nanowires, Nano Lett. 12, 4437 (2012).

[7] F. Meier and B. P. Zachachrenya, Optical Orientation: Modern Problems in Condensed Matter Science (NorthHolland, Amsterdam, 1984), Vol. 8.

[8] Y. K. Kato, R. C. Myers, A.C. Gossard, and D. D. Awschalom, Coherent Spin Manipulation without Magnetic Fields in Strained Semiconductors, Nature (London) 427, 50 (2004).

[9] Y. Kato, R. C. Myers, A. C. Gossard, and D. D. Awschalom, Electrical Initialization and Manipulation of Electron Spins in an L-Shaped Strained n-InGaAs Channel, Appl. Phys. Lett. 87, 022503 (2005).

[10] S. A. Crooker and D. L. Smith, Imaging Spin Flows in Semiconductors Subject to Electric, Magnetic, and Strain Fields, Phys. Rev. Lett. 94, 236601 (2005).

[11] Y. Yafet, $g$ Factors and Spin-Lattice Relaxation of Conduction Electrons, Solid State Phys. 14, 1 (1963).

[12] M. I. D'yakonov and V.I. Perel', Spin Relaxation of Conduction Electrons in Noncentrosymmetric Semiconductors, Sov. Phys. Solid State 13, 3023 (1972).

[13] W. H. Lau, J. T. Olesberg, and M.E. Flatté, Electron-Spin Decoherence in Bulk and Quantum-Well Zinc-Blende Semiconductors, Phys. Rev. B 64, 161301 (2001).

[14] M. I. D'yakonov and V. Y. Kachorovskii, Spin Relaxation of Two-Dimensional Electrons in Nncentrosymmetric Semiconductors, Sov. Phys. Semicond. 20, 110 (1986).
[15] E. L. Ivchenko and G. E. Pikus, Superlattices and Other Heterostructures (Springer, New York, 1997).

[16] A. Bournel, P. Dollfus, P. Bruno, and P. Hesto, GateInduced Spin Precession in an $\mathrm{In}_{0.53} \mathrm{Ga}_{0.47} \mathrm{As}$ TwoDimensional Electron Gas, Eur. Phys. J. Appl. Phys. 4, 1 (1998).

[17] A. G. Mal'shukov and K. A. Chao, Waveguide Diffusion Modes and Slowdown of D'yakonov-Perel' Spin Relaxation in Narrow Two-Dimensional Semiconductor Channels, Phys. Rev. B 61, R2413 (2000).

[18] A. A. Kiselev and K. W. Kim, Progressive Suppression of Spin Relaxation in Two-Dimensional Channels of Finite Width, Phys. Rev. B 61, 13115 (2000).

[19] T. P. Pareek and P. Bruno, Spin Coherence in a TwoDimensional Electron Gas with Rashba Spin-Orbit Interaction, Phys. Rev. B 65, 241305 (2002).

[20] A. W. Holleitner, V. Sih, R. C. Myers, A. C. Gossard, and D. D. Awschalom, Suppression of Spin Relaxation in Submicron InGaAs Wires, Phys. Rev. Lett. 97, 036805 (2006).

[21] P. Schwab, M. Dzierzawa, C. Gorini, and R. Raimondi, Spin Relaxation in Narrow Wires of a Two-Dimensional Electron Gas, Phys. Rev. B 74, 155316 (2006).

[22] C.-H. Chang, J. Tsai, H.-F. Lo, and A. G. Mal'shukov, Semiclassical Path Integral Approach for Spin Relaxations in Narrow Wires, Phys. Rev. B 79, 125310 (2009).

[23] V. A. Froltsov, Diffusion of Inhomogeneous Spin Distribution in a Magnetic Field Parallel to Interfaces of a III-V Semiconductor Quantum Well, Phys. Rev. B 64, 045311 (2001).

[24] Y. V. Pershin, Long-Lived Spin-Coherence States in Semiconductor Heterostructures, Phys. Rev. B 71, 155317 (2005).

[25] F. E. Meijer, A. F. Morpurgo, T. M. Klapwijk, T. Koga, and J. Nitta, Competition between Spin-Orbit Interaction and Zeeman Coupling in Rashba Two-Dimensional Electron Gases, Phys. Rev. B 70, 201307 (2004).

[26] S. M. Frolov, S. Lüscher, W. Yu, Y. Ren, J. A. Folk, and W. Wegscheider, Ballistic Spin Resonance, Nature (London) 458, 868 (2009).

[27] S. Lüscher, S. M. Frolov, and J. A. Folk, Numerical Study of Resonant Spin Relaxation in Quasi-One-Dimensional Channels, Phys. Rev. B 82, 115304 (2010).

[28] É.I. Rashba, Properties of Semiconductors with an Extremum Loop I. Cyclotron and Combinational Resonance in a Magnetic Field Perpendicular to the Plane of the Loop, Sov. Phys. Solid State 2, 1109 (1960).

[29] Y. A. Bychkov and É. I. Rashba, Oscillatory Effects and the Magnetic Susceptibility of Carriers in Inversion Layers, J. Phys. C 17, 6039 (1984).

[30] G. Dresselhaus, Spin-Orbit Coupling Effects in zinc-Blende Structures, Phys. Rev. 100, 580 (1955).

[31] M. Johnson and R. H. Silsbee, Interfacial Charge-Spin Coupling: Injection and Detection of Spin Magnetization in Metals, Phys. Rev. Lett. 55, 1790 (1985).

[32] F. J. Jedema, H. B. Heersche, A. T. Filip, J. J. A. Baselmans, and B. J. van Wees, Electrical Detection of Spin Precession in a Metallic Mesoscopic Spin Valve, Nature (London) 416, 713 (2002). 
[33] S. M. Frolov, A. Venkatesan, W. Yu, J. A. Folk, and W. Wegscheider, Electrical Generation of Pure Spin Currents in a Two-Dimensional Electron Gas, Phys. Rev. Lett. 102, 116802 (2009).

[34] A. M. Finkel'stein, Weak Localization and Coulomb Interaction in Disordered Systems Z. Phys. B 56, 189 (1984).

[35] P. Wölfle and R. N. Bhatt, Electron Localization in Anisotropic Systems, Phys. Rev. B 30, 3542 (1984).

[36] A. G. Mal'shukov, K. A. Chao, and M. Willander, Weak Localization Corrections to Spin and Particle Transport in Semiconductor Quantum Wells. Effects of the Spin-Orbit Interaction, Phys. Scr. T66, 138 (1996).
[37] D. Forster, Hydrodynamic Fluctuations, Broken Symmetry, and Correlation Functions (Benjamin, Reading, MA, 1975).

[38] S. A. Crooker, M. Furis, X. Lou, C. Adelmann, D. L. Smith, C. J. Palmstrøm, and P. A. Crowell, Imaging Spin Transport in Lateral Ferromagnet/Semiconductor Structures, Science 309, 2191 (2005).

[39] H. C. Koo, J. H. Kwon, J. Eom, J. Chang, S. H. Han, and M. Johnson, Control of Spin Precession in a Spin-Injected Field-Effect Transistor, Science 325, 1515 (2009).

[40] M. O. Hachiya, G. Usaj, and J. C. Egues, Ballistic Spin Resonance in Multisubband Quantum Wires, Phys. Rev. B 89, 125310 (2014). 